\title{
Stability Analysis for Parameterized Variational Systems with Implicit Constraints
}

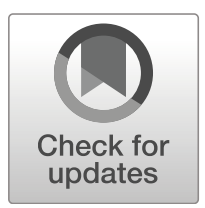

\author{
Matúš Benko ${ }^{1} \cdot$ Helmut Gfrerer ${ }^{1}$ (D) . Jiří V. Outrata ${ }^{2,3}$
}

Received: 17 December 2018 / Accepted: 13 June 2019 / Published online: 3 July 2019

(C) The Author(s) 2019

\begin{abstract}
In the paper we provide new conditions ensuring the isolated calmness property and the Aubin property of parameterized variational systems with constraints depending, apart from the parameter, also on the solution itself. Such systems include, e.g., quasi-variational inequalities and implicit complementarity problems. Concerning the Aubin property, possible restrictions imposed on the parameter are also admitted. Throughout the paper, tools from the directional limiting generalized differential calculus are employed enabling us to impose only rather weak (non- restrictive) qualification conditions. Despite the very general problem setting, the resulting conditions are workable as documented by some academic examples.
\end{abstract}

Keywords Parameterized variational system · Solution map · Aubin property · Isolated calmness property

Mathematics Subject Classification (2010) 49J53 • 90C31 · 90C46

Helmut Gfrerer

helmut.gfrerer@jku.at

Matúš Benko

benko@numa.uni-linz.ac.at

Jiří V. Outrata

outrata@utia.cas.cz

1 Institute of Computational Mathematics, Johannes Kepler University Linz, A-4040 Linz, Austria

2 Institute of Information Theory Automation, Academy of Sciences of the Czech Republic, 18208 Prague, Czech Republic

3 Centre for Informatics Applied Optimization, Federation University of Australia, POB 663, Ballarat, Vic 3350, Australia 


\section{Introduction}

In variational analysis, a great effort has been devoted to the study of stability and sensitivity of solution maps to parameter-dependent optimization and equilibrium problems. In particular, the researchers have investigated various Lipschitzian properties of these maps around given reference points. To obtain useful results, one employs typically some efficient tools of generalized differentiation discussed in a detailed way in the monographs [5, 25, 28, 30, 34]. Starting from 2011, the available arsenal of these tools includes also the calculus of directional limiting normal cones and coderivatives which enables us in some cases a finer analysis of parametric equilibria than its non-directional counterpart. This new theory has been initiated in [19] and then thoroughly developed in a number of papers authored and co-authored by H. Gfrerer [1, 9-13, 15, 16, 18].

In particular, in [16] one finds rather weak (non-restrictive) conditions ensuring the calmness and the Aubin property of general implicitly defined multifunctions. The criterion for the Aubin property has then been worked out in [17] for a class of parametric variational systems with fixed (non-perturbed) constraint sets and in [18] for systems with implicit parameter-dependent constraints. The model from [18] was investigated already in [29] by using the (classical) generalized differential calculus of B. Mordukhovich. It encompasses quasi-variational inequalities (QVIs), implicit complementarity problems and also standard variational inequalities of the first kind with parameter-dependent constraints.

In this paper we consider the same model as in [29] and [18] but remove the (rather severe) non-degeneracy-type assumption imposed in [18] on the constraint system. Instead of it, we make use of a (much weaker) metric inequality stated in Assumption 1. Further, we analyze now not just the standard Aubin property of the considered solution map, denoted by $S$, but the Aubin property relative to a given set of feasible parameters. Clearly, $\mathrm{S}$ may enjoy this type of Lipschitzian stability even when the standard Aubin property is violated. Finally, we provide in this paper also a new condition, ensuring the isolated calmness of $S$.

The structure of the considered constraint system has enabled us to employ some strong results from [4,5] and [16] concerning tangents and normals to the graph of the normalcone mapping associated with a convex polyhedral set. More precisely, these tangents and normals can be expressed via some faces of an associated critical cone. This representation substantially contributes to the workability of final conditions ensuring the Aubin property of $S$. In addition, also some other statements in connection with directional non-degeneracy and directional metric regularity could be formulated in terms of these faces.

The plan of the paper is as follows. Sections 2.1 and 2.2 provide the reader with basic notions of the standard and directional generalized differential calculus and with some basic facts about those Lipschitzian stability properties which are extensively used throughout the whole paper. Section 2.3 contains the necessary background from the theory of convex polyhedral sets and polyhedral multifunctions. The last preliminary Section 2.4 is then devoted to the directional metric subregularity of a particular multifunction, which arises later as a qualification condition, and to the new notion of directional non-degeneracy of a constraint system, playing a central role in the subsequent development. Section 3 concerns the general model of an implicitly defined multifunction considered in [16]. In this framework we find there a directional variant of the Levy-Rockafellar characterization of the isolated calmness property and a counterpart of [16, Theorem 4.4] corresponding to the Aubin property relative to a set of feasible parameters. In the rest of the paper these statements are worked out for the considered variational system with implicit constraints. So, in Section 4 the respective graphical derivative is computed, which is a basis for the formulation of the final condition ensuring the isolated calmness property of $S$ presented in 
Section 5. Therafter, in Section 6 one finds a new workable sufficient condition guaranteeing the Aubin property of $\mathrm{S}$ relative to a given set of feasible parameters. Both these final results as well as some other important statements are illustrated by examples.

There are well-known equilibria in economy and mechanics modeled by QVIs and implicit complementarity problems, cf. [2]. As an example, let us mention the generalized Nash equilibrium problems (GNEPs) which describe, e.g., the behavior of agents acting on markets with a limited amount of resources. Very often, these equilibria depend on some uncertain data which can be viewed as parameters. The results of this paper can then be used in post-optimal analysis of such equilibria, where the stability issues are of ultimate importance.

Given a set-valued mapping $M: \mathbb{R}^{l} \times \mathbb{R}^{n} \rightrightarrows \mathbb{R}^{m}$, the general implicitly defined multifunction analyzed in [16] is given by the relation

$$
0 \in M(p, x) .
$$

We are going to analyze the associated solution mapping $S: \mathbb{R}^{l} \rightrightarrows \mathbb{R}^{n}$ defined by

$$
S(p):=\left\{x \in \mathbb{R}^{n} \mid 0 \in M(p, x)\right\} .
$$

The variational system investigated in [29] and [18] attains the form

$$
0 \in M(p, x):=f(p, x)+\widehat{N}_{\Gamma(p, x)}(x) \text { with } \Gamma(p, x):=\{z \mid g(p, x, z) \in D\},
$$

where $f: \mathbb{R}^{l} \times \mathbb{R}^{n} \rightarrow \mathbb{R}^{n}$ is continuously differentiable, $g: \mathbb{R}^{l} \times \mathbb{R}^{n} \times \mathbb{R}^{n} \rightarrow \mathbb{R}^{s}$ is twice continuously differentiable and $D \subset \mathbb{R}^{s}$ is a convex polyhedral set.

The following notation is employed. Given a set $A \subset \mathbb{R}^{n}, \operatorname{sp} A$ stands for the linear hull of $A$, ri $A$ is the relative interior of $A$ and $A^{\circ}$ is the (negative) polar of $A$. We denote by $\operatorname{dist}(x, A):=\inf _{y \in A}\|x-y\|$ the usual point to set distance with the convention $\operatorname{dist}(x, \emptyset)=$ $\infty$. For a sequence $x_{k}, x_{k} \stackrel{A}{\rightarrow} \bar{x}$ stands for $x_{k} \rightarrow \bar{x}$ with $x_{k} \in A$. For a convex cone $K$, lin $K$ denotes the lineality space of $K$, i.e., the set $K \cap(-K)$. Further, $\mathscr{B}_{\mathbb{R}^{n}}, \mathscr{S}_{\mathbb{R}^{n}}$ is the unit ball and the unit sphere in $\mathbb{R}^{n}$, respectively. Given a vector $a \in \mathbb{R}^{n},[a]$ is the linear space generated by $a$ and $[a]^{\perp}$ stands for the orthogonal complement to $[a]$. Finally, given a setvalued map $F: \mathbb{R}^{n} \rightrightarrows \mathbb{R}^{m}$, gph $F:=\left\{(x, y) \in \mathbb{R}^{n} \times \mathbb{R}^{m} \mid y \in F(x)\right\}$ stands for the graph of $F$ and Lim $\sup _{x \rightarrow \bar{x}} F(x)$ denotes the outer set limit in the sense of Painlevé-Kuratowski.

\section{Preliminaries}

\subsection{Variational Geometry and Generalized Differentiation}

We start by recalling several definitions and results from variational analysis. Let $\Omega \subset \mathbb{R}^{n}$ be an arbitrary closed set and $\bar{x} \in \Omega$. The contingent (also called Bouligand or tangent) cone to $\Omega$ at $\bar{x}$, denoted by $T_{\Omega}(\bar{x})$, is given by

$$
T_{\Omega}(\bar{x}):=\left\{u \in \mathbb{R}^{n} \mid \exists\left(u_{k}\right) \rightarrow u,\left(t_{k}\right) \downarrow 0: \bar{x}+t_{k} u_{k} \in \Omega \forall k\right\} .
$$

A tangent $u \in T_{\Omega}(\bar{x})$ is called derivable if $\operatorname{dist}(\bar{x}+t u, \Omega)=o(t)$.

We denote by

$$
\widehat{N}_{\Omega}(\bar{x}):=T_{\Omega}(\bar{x})^{\circ}\left\{x^{*} \in \mathbb{R}^{n} \mid \limsup _{x^{\prime} \stackrel{\Omega}{\rightarrow} \bar{x}} \frac{\left\langle x^{*}, x^{\prime}-\bar{x}\right\rangle}{\left\|x^{\prime}-\bar{x}\right\|} \leq 0\right\}
$$


the Fréchet (regular) normal cone to $\Omega$ at $\bar{x}$. The limiting (Mordukhovich) normal cone to $\Omega$ at $\bar{x}$ is defined by

$$
N_{\Omega}(\bar{x}):=\left\{x^{*} \in \mathbb{R}^{n} \mid \exists\left(x_{k}\right) \stackrel{\Omega}{\rightarrow} \bar{x},\left(x_{k}^{*}\right) \rightarrow x^{*}: x_{k}^{*} \in \widehat{N}_{\Omega}\left(x_{k}\right) \forall k\right\} .
$$

Finally, given a direction $u \in \mathbb{R}^{n}$, we denote by

$$
N_{\Omega}(\bar{x} ; u):=\underset{\substack{t \downarrow \operatorname{sim} \\ u^{\prime} \rightarrow u}}{ } \widehat{N}_{\Omega}\left(\bar{x}+t u^{\prime}\right)
$$

the directional limiting normal cone to $\Omega$ in direction $u$ at $\bar{x}$.

If $\bar{x} \notin \Omega$, we put $T_{\Omega}(\bar{x})=\emptyset, \widehat{N}_{\Omega}(\bar{x})=\emptyset, N_{\Omega}(\bar{x})=\emptyset$ and $N_{\Omega}(\bar{x} ; u)=\emptyset$. Further note that $N_{\Omega}(\bar{x} ; u)=\emptyset$ whenever $u \notin T_{\Omega}(\bar{x})$. If $\Omega$ is convex, then $\widehat{N}_{\Omega}(\bar{x})=N_{\Omega}(\bar{x})$ amounts to the classical normal cone in the sense of convex analysis and we will write $N_{\Omega}(\bar{x})$.

Given a pair $\left(\bar{x}, \bar{x}^{*}\right) \in \operatorname{gph} \widehat{N}_{\Omega}$ we denote by

$$
\mathscr{K}_{\Omega}\left(\bar{x}, \bar{x}^{*}\right):=T_{\Omega}(\bar{x}) \cap\left[\bar{x}^{*}\right]^{\perp}
$$

the critical cone to $\Omega$ at $\bar{x}$ with respect to $\bar{x}^{*}$.

The following generalized derivatives of set-valued mappings are defined by means of the tangent cone and the (directional) limiting normal cone to the graph of the mapping.

Definition 2.1 Let $F: \mathbb{R}^{n} \rightrightarrows \mathbb{R}^{m}$ be a set-valued mapping having locally closed graph around $(\bar{x}, \bar{y}) \in \operatorname{gph} F$.

(i) The set-valued map $D F(\bar{x}, \bar{y}): \mathbb{R}^{n} \rightrightarrows \mathbb{R}^{m}$, defined by

$$
D F(\bar{x}, \bar{y})(u):=\left\{v \in \mathbb{R}^{m} \mid(u, v) \in T_{\operatorname{gph} F}(\bar{x}, \bar{y})\right\}, u \in \mathbb{R}^{n}
$$

is called the graphical derivative of $F$ at $(\bar{x}, \bar{y})$.

(ii) The set-valued map $\widehat{D}^{*} F(\bar{x}, \bar{y}): \mathbb{R}^{m} \rightrightarrows \mathbb{R}^{n}$

$$
\widehat{D}^{*} F(\bar{x}, \bar{y})\left(v^{*}\right):=\left\{u^{*} \in \mathbb{R}^{n} \mid\left(u^{*},-v^{*}\right) \in \widehat{N}_{\mathrm{gph} F}(\bar{x}, \bar{y})\right\}, v^{*} \in \mathbb{R}^{m}
$$

is called the regular (Fréchet) coderivative of $F$ at $(\bar{x}, \bar{y})$.

(iii) The set-valued map $D^{*} F(\bar{x}, \bar{y}): \mathbb{R}^{m} \rightrightarrows \mathbb{R}^{n}$, defined by

$$
D^{*} F(\bar{x}, \bar{y})\left(v^{*}\right):=\left\{u^{*} \in \mathbb{R}^{n} \mid\left(u^{*},-v^{*}\right) \in N_{\operatorname{gph} F}(\bar{x}, \bar{y})\right\}, v^{*} \in \mathbb{R}^{m}
$$

is called the limiting (Mordukhovich) coderivative of $F$ at $(\bar{x}, \bar{y})$.

(iv) Given a pair of directions $(u, v) \in \mathbb{R}^{n} \times \mathbb{R}^{m}$, the set-valued map $D^{*} F((\bar{x}, \bar{y}) ;(u, v))$ : $\mathbb{R}^{m} \rightrightarrows \mathbb{R}^{n}$, defined by

$$
D^{*} F((\bar{x}, \bar{y}) ;(u, v))\left(v^{*}\right):=\left\{u^{*} \in \mathbb{R}^{n} \mid\left(u^{*},-v^{*}\right) \in N_{\operatorname{gph} F}((\bar{x}, \bar{y}) ;(u, v))\right\}, v^{*} \in \mathbb{R}^{m}
$$

is called the directional limiting coderivative of $F$ in direction $(u, v)$ at $(\bar{x}, \bar{y})$.

\subsection{Regularity and Lipschitzian Properties of Set-Valued Mappings}

First we recall some well-known definitions.

Definition 2.2 Let $F: \mathbb{R}^{n} \rightrightarrows \mathbb{R}^{m}$ be a mapping and let $(\bar{x}, \bar{y}) \in \operatorname{gph} F$. We say that $F$ is metrically regular around $(\bar{x}, \bar{y})$ if there are neighborhoods $U$ of $\bar{x}$ and $V$ of $\bar{y}$ along with some real $\kappa \geq 0$ such that

$$
\operatorname{dist}\left(x, F^{-1}(y)\right) \leq \kappa \operatorname{dist}(y, F(x)) \forall(x, y) \in U \times V .
$$


When fixing $y=\bar{y}$ in this condition, $F$ is said to be metrically subregular at $(\bar{x}, \bar{y})$, i.e., we require

$$
\operatorname{dist}\left(x, F^{-1}(\bar{y})\right) \leq \kappa \operatorname{dist}(\bar{y}, F(x)) \forall x \in U \text {. }
$$

A well-known coderivative characterization of metric regularity is known as "Mordukhovich criterion" and reads as follows.

Theorem 2.3 ([30, Theorem 3.3]) Assume that the set-valued mapping $F: \mathbb{R}^{n} \rightrightarrows \mathbb{R}^{m}$ has locally closed graph around $(\bar{x}, \bar{y}) \in \operatorname{gph} F$. Then $F$ is metrically regular around $(\bar{x}, \bar{y})$ if and only if

$$
0 \in D^{*} F(\bar{x}, \bar{y})\left(v^{*}\right) \Rightarrow v^{*}=0 .
$$

One can find numerous sufficient conditions for metric subregularity in the literature, see, e.g., [7-12, 20, 24, 26, 35]. However, these sufficient conditions are often very difficult to verify. The following sufficient condition for metric subregularity is not as week as possible but it is stable with respect to certain perturbations, cf. [6].

Theorem 2.4 ([16, Theorem 2.6]) Assume that the set-valued mapping $F: \mathbb{R}^{n} \rightrightarrows \mathbb{R}^{m}$ has locally closed graph around $(\bar{x}, \bar{y}) \in \mathrm{gph} F$. If

$$
\forall 0 \neq u \in \mathbb{R}^{n}: 0 \in D^{*} F((\bar{x}, \bar{y}) ;(u, 0))\left(v^{*}\right) \Rightarrow v^{*}=0,
$$

then $F$ is metrically subregular at $(\bar{x}, \bar{y})$.

In order to define a directional version of metric (sub)regularity, consider for a direction $u \in \mathbb{R}^{n}$ and positive reals $\rho, \delta$ the set

$$
\mathscr{V}_{\rho, \delta}(u):=\left\{d \in \rho \mathscr{B}_{\mathbb{R}^{n}} \mid\|\| u\|d-\| d\|u\| \leq \delta\|u\|\|d\|\right\} .
$$

We say that $\mathscr{V}$ is a directional neighborhood of $u$ if $\mathscr{V}_{\rho, \delta}(u) \subset \mathscr{V}$ for some $\rho, \delta>0$.

Definition 2.5 Let $F: \mathbb{R}^{n} \rightrightarrows \mathbb{R}^{m}$ be a mapping and let $(\bar{x}, \bar{y}) \in \operatorname{gph} F$.

1. Given a direction $u \in \mathbb{R}^{n}$ we say that $F$ is metrically subregular in direction $u$ at $(\bar{x}, \bar{y})$ if (2.5) holds with $\bar{x}+\mathscr{U}$ in place of $U$, where $\mathscr{U}$ is a directional neighborhood of $u$.

2. Given a direction $(u, v) \in \mathbb{R}^{n} \times \mathbb{R}^{m}$ we say that $F$ is metrically regular in direction $(u, v)$ at $(\bar{x}, \bar{y})$ if there is a directional neighborhoods $\mathscr{W}$ of $(u, v)$ together with reals $\kappa \geq 0$ and $\delta>0$ such that (2.4) holds for all $(x, y) \in(\bar{x}, \bar{y})+\mathscr{W}$ satisfying $\|(u, v)\| \operatorname{dist}((x, y), \operatorname{gph} F) \leq \delta\|(u, v)\|\|(x, y)-(\bar{x}, \bar{y})\|$.

If a mapping $F$ is metrically regular in direction $(u, 0)$ at $(\bar{x}, \bar{y})$ then it is also metrically subregular in direction $u$, cf. [10, Lemma 1]. Further note that a mapping is always metrically regular in a direction $(u, v)$ at $(\bar{x}, \bar{y})$ whenever $(u, v) \notin T_{\operatorname{gph} F}(\bar{x}, \bar{y})$, i.e., $v \notin D F(\bar{x}, \bar{y})(u)$. Similarly, if $0 \notin D F(\bar{x}, \bar{y})(u)$, then $F$ is metrically subregular in direction $u$ at $(\bar{x}, \bar{y})$.

Theorem 2.6 Assume that the set-valued mapping $F: \mathbb{R}^{n} \rightrightarrows \mathbb{R}^{m}$ has locally closed graph around $(\bar{x}, \bar{y}) \in \operatorname{gph} F$ and let $u \in \mathbb{R}^{n}$ be given. Then $F$ is metrically regular in direction $(u, 0)$ at $(\bar{x}, \bar{y})$ if and only if

$$
0 \in D^{*} F((\bar{x}, \bar{y}) ;(u, 0))\left(v^{*}\right) \Rightarrow v^{*}=0 .
$$


Proof Follows from [10, Theorem 5].

Comparing Definition 2.5 with Definition 2.2 we see that metric regularity around $(\bar{x}, \bar{y})$ is equivalent with metric regularity in direction $(0,0)$ at $(\bar{x}, \bar{y})$. This is reflected also in conditions (2.6) and (2.7) with $u=0$. Further note that the sufficient condition for metric subregularity of Theorem 2.4 says that mapping $F$ is metrically regular at $(\bar{x}, \bar{y})$ in every direction $(u, 0)$ with $u \neq 0$.

The following notion of stability was introduced by Robinson [31].

Definition 2.7 Consider the system

$$
h(p, x) \in C
$$

for a mapping $h: P \times \mathbb{R}^{n} \rightarrow \mathbb{R}^{m}$ and a set $C \subset \mathbb{R}^{m}$, where $P$ is a topological space and denote

$$
S(p):=\left\{x \in \mathbb{R}^{n} \mid h(p, x) \in C\right\}, p \in P .
$$

We say that the system (2.8) enjoys the Robinson stability property at $(\bar{p}, \bar{x}) \in \operatorname{gph} S$ if there are neighborhoods $Q$ of $\bar{p}, U$ of $\bar{x}$ and a real $\kappa \geq 0$ such that

$$
\operatorname{dist}(x, S(p)) \leq \kappa \operatorname{dist}(h(p, x), C) \forall(p, x) \in Q \times U .
$$

Comparing the definition of Robinson stability with that of metric regularity we see that in case when $P=\mathbb{R}^{l}$ and $h$ is of the form $h(p, x)=\tilde{h}(x)-p$, the property of Robinson stability of $(2.8)$ at $(\bar{p}, \bar{x})$ is equivalent to metric regularity of the mapping $\tilde{h}(\cdot)-C$ around $(\bar{x}, \bar{p})$. For sufficient conditions for Robinson stability we refer to the recent paper [14]. Here we mention only the following result.

Theorem 2.8 Let $(\bar{p}, \bar{x}) \in h^{-1}(C)$ be given and assume that $h$ is differentiable with respect to the second component and both $h$ and $\nabla_{2} h$ are continuous, whereas $C$ is closed. If

$$
\nabla_{2} h(\bar{p}, \bar{x})^{T} \mu=0, \mu \in N_{C}(h(\bar{p}, \bar{x})) \Rightarrow \mu=0,
$$

then the system (2.8) enjoys the Robinson stability property at $(\bar{p}, \bar{x})$.

Proof Follows immediately from [14, Corollary 3.6].

We now turn to Lipschitzian properties of set-valued mappings.

Definition 2.9 Let $S: \mathbb{R}^{m} \rightrightarrows \mathbb{R}^{n}$ be a set-valued map and let $(\bar{y}, \bar{x}) \in \operatorname{gph} S$.

1. $S$ is called to be calm at $(\bar{y}, \bar{x})$ if there is a neighborhood $U$ of $\bar{x}$ together with a real $L \geq 0$ such that

$$
S(y) \cap U \subset S(\bar{y})+L\|y-\bar{y}\| \mathscr{B}_{\mathbb{R}^{n}} \forall y \in \mathbb{R}^{m} .
$$

If, in addition, $S(\bar{y})=\{\bar{x}\}$ is a singleton we say that $S$ has the isolated calmness property at $(\bar{y}, \bar{x})$.

2. Given a set $Y \subset \mathbb{R}^{m}$ containing $\bar{y}$, the mapping $S$ is said to have the Aubin property relative to $Y$ around $(\bar{y}, \bar{x})$ if there are neighborhoods $V$ of $\bar{y}, U$ of $\bar{x}$ and a real $L \geq 0$ such that

$$
S(y) \cap U \subset S\left(y^{\prime}\right)+L\left\|y-y^{\prime}\right\| \mathscr{B}_{\mathbb{R}^{n}} \forall y, y^{\prime} \in Y \cap V .
$$

This condition with $V$ in place of $Y \cap V$ is simply the Aubin propery around $(\bar{y}, \bar{x})$. 
It is well-known [4] that $F$ is metrically subregular at $(\bar{x}, \bar{y})$ if and only if its inverse mapping $F^{-1}$ is calm at $(\bar{y}, \bar{x})$. Further, metric regularity is equivalent with the Aubin property of the inverse mapping.

\subsection{Polyhedral Sets}

Recall that a set $D \subset \mathbb{R}^{s}$ is said to be convex polyhedral if it can be represented as the intersection of finitely many halfspaces. We say that a set $E \subset \mathbb{R}^{s}$ is polyhedral if it is the union of finitely many convex polyhedral sets. If a set $E$ is polyhedral, then for every $\bar{z} \in E$ there is some neighborhood $W$ of $\bar{z}$ such that

$$
(E-\bar{z}) \cap W=T_{E}(\bar{z}) \cap W .
$$

Given a convex polyhedral set $D$ and a point $\bar{z} \in D$, then the tangent cone $T_{D}(\bar{z})$ and the normal cone $N_{D}(\bar{z})$ are convex polyhedral cones and there is a neighborhood $W$ of $\bar{z}$ such that

$$
T_{D}(z)=T_{D}(\bar{z})+[z-\bar{z}] \supset T_{D}(\bar{z}), N_{D}(z)=N_{D}(\bar{z}) \cap[z-\bar{z}]^{\perp} \subset N_{D}(\bar{z}) \forall z \in D \cap W .
$$

The graph of the normal cone mapping to $D$ is a polyhedral set and for every pair $\left(z, z^{*}\right) \in$ gph $N_{D}$ we have

$$
T_{\operatorname{gph} N_{D}}\left(z, z^{*}\right)=\operatorname{gph} N_{\mathscr{K}_{D}\left(z, z^{*}\right)},
$$

see, e.g., [5, Lemma 2E.4].

For two convex polyhedral cones $K_{1}, K_{2} \subset \mathbb{R}^{s}$ their polars as well as their sum $K_{1}+K_{2}$ and their intersection $K_{1} \cap K_{2}$ are again convex polyhedral cones and

$$
\left(K_{1}+K_{2}\right)^{\circ}=K_{1}^{\circ} \cap K_{2}^{\circ},\left(K_{1} \cap K_{2}\right)^{\circ}=K_{1}^{\circ}+K_{2}^{\circ} .
$$

For a convex polyhedral cone $K \subset \mathbb{R}^{s}$ and a point $z \in K$ we have

$$
T_{K}(z)=K+[z], N_{K}(z)=K^{\circ} \cap[z]^{\perp} .
$$

A face $\mathscr{F}$ of $K$ can always be written in the form

$$
\mathscr{F}=K \cap\left[z^{*}\right]^{\perp}
$$

for some $z^{*} \in K^{\circ}$. The cone $K$ has the representation

$$
K=\left\{z \in \mathbb{R}^{s} \mid a_{i}^{T} z=0, i \in \bar{J}, a_{i}^{T} z \leq 0 i \in \bar{I} \backslash \bar{J}\right\},
$$

where $\bar{J} \subset \bar{I}$ are two finite index sets and $a_{i} \in \mathbb{R}^{s}, i \in \bar{I}$. By enlarging $\bar{J}$ when necessary we can assume that there exists some $z_{0}$ such that $a_{i}^{T} z_{0}=0, i \in \bar{J}, a_{i}^{T} z_{0}<0, i \in \bar{I} \backslash \bar{J}$. Then a subset $\mathscr{F} \subset K$ is a face if and only if there is some index set $J, \bar{J} \subset J \subset \bar{I}$ such that

$$
\mathscr{F}=\left\{z \in \mathbb{R}^{s} \mid a_{i}^{T} z=0, i \in J, a_{i}^{T} z \leq 0 i \in \bar{I} \backslash J\right\} .
$$

By possibly enlarging $J$ we can find a unique index set, denoted by $J_{\mathscr{F}}$, such that

$$
\text { ri } \mathscr{F}=\left\{z \in \mathbb{R}^{s} \mid a_{i}^{T} z=0, i \in J_{\mathscr{F}}, a_{i}^{T} z<0 i \in \bar{I} \backslash J_{\mathscr{F}}\right\} .
$$

It follows that

$$
\mathscr{F}-\mathscr{F}=\left\{z \in \mathbb{R}^{s} \mid a_{i}^{T} z=0, i \in J_{\mathscr{F}}\right\}
$$




\subsection{Directional Non-Degeneracy}

In what follows the property of directional metric (sub)regularity of a particular mapping will play an important role. Let $D \subset \mathbb{R}^{s}$ be a convex polyhedral set, let $\tilde{g}: \mathbb{R}^{m} \rightarrow \mathbb{R}^{s}$ be continuously differentiable and consider the mapping $F: \mathbb{R}^{m} \times \mathbb{R}^{s} \rightrightarrows \mathbb{R}^{s} \times \mathbb{R}^{s}$ given by

$$
F(y, \lambda):=(\tilde{g}(y), \lambda)-\operatorname{gph} N_{D} .
$$

Given some point $(\bar{y}, \lambda) \in F^{-1}(0)$ and some direction $(v, \eta) \in \mathbb{R}^{m} \times \mathbb{R}^{s}$, we want to investigate metric subregularity of $F$ in direction $(v, \eta)$ at $(\bar{y}, \lambda)$, in particular when $v \neq 0$. We denote

$$
\Theta(\bar{y}, v):=\left\{(\lambda, \eta) \in N_{D}(\tilde{g}(\bar{y})) \times \mathbb{R}^{s} \mid(\nabla \tilde{g}(\bar{y}) v, \eta) \in \operatorname{gph} N_{\mathscr{K}_{D}(\tilde{g}(\bar{y}), \lambda)}\right\},(\bar{y}, v) \in \tilde{g}^{-1}(D) \times \mathbb{R}^{m} .
$$

Recall that $F$ is by definition metrically subregular in direction $(v, \eta)$ at $((\bar{y}, \lambda),(0,0))$ whenever

$(0,0) \notin D F((\bar{y}, \lambda),(0,0))(v, \eta)=(\nabla \tilde{g}(\bar{y}) v, \eta)-T_{\operatorname{gph} N_{D}}(\tilde{g}(\bar{y}), \lambda) \Leftrightarrow(\nabla \tilde{g}(\bar{y}) v, \eta) \notin T_{\mathrm{gph} N_{D}}(\tilde{g}(\bar{y}), \lambda)$, i.e., taking into account (2.9), whenever $(\lambda, \eta) \notin \Theta(\bar{y}, v)$.

In our analysis we restrict ourselves to the characterization of metric regularity of $F$ in directions $((v, \eta),(0,0))$ which implies metric subregularity of $F$ in direction $(v, \eta)$. The following lemma is a slight generalization of [18, Proposition 2].

Lemma 2.10 Let $\bar{y} \in \tilde{g}^{-1}(D), v \in \mathbb{R}^{m}$ and $(\lambda, \eta) \in \Theta(\bar{y}, v)$ be given. Then the mapping $F$ defined in (2.12) is metrically regular in direction $((v, \eta),(0,0))$ at $((\bar{y}, \lambda),(0,0))$ if and only if for every face $\mathscr{F}$ of the critical cone $\mathscr{K}_{D}(\tilde{g}(\bar{y}), \lambda)$ with $\nabla \tilde{g}(\bar{y})(v) \in \mathscr{F} \subset[\eta]^{\perp}$ one has

$$
\nabla \tilde{g}(\bar{y})^{T} \mu=0, \mu \in(\mathscr{F}-\mathscr{F})^{\circ} \Rightarrow \mu=0 .
$$

Proof The characterization (3.23) reads in our special case as

$$
\left(\nabla \tilde{g}(\bar{y})^{T} \mu, \xi\right)=(0,0),(\mu, \xi) \in N_{\mathrm{gph} N_{D}}((\tilde{g}(\bar{y}), \lambda),(\nabla \tilde{g}(\bar{y}) v, \eta)) \Rightarrow(\mu, \xi)=(0,0),
$$

see also $\left[13\right.$, Theorem 1]. By [16, Theorem 2.12], $N_{\operatorname{gph} N_{D}}((\tilde{g}(\bar{y}), \lambda),(\nabla \tilde{g}(\bar{y}) v, \eta))$ amounts to the union of all product sets $K^{\circ} \times K$ associated with cones $K$ of the form $\mathscr{F}_{1}-\mathscr{F}_{2}$, where $\mathscr{F}_{1}, \mathscr{F}_{2}$ are faces of the critical cone $\mathscr{K}_{D}(\tilde{g}(\bar{y}), \lambda)$ with $\nabla \tilde{g}(\bar{y})(v) \in \mathscr{F}_{2} \subset \mathscr{F}_{1} \subset[\eta]^{\perp}$. Thus, by Theorem 2.6 the claimed directional metric regularity is equivalent to the condition that the implication

$$
\nabla \tilde{g}(\bar{y})^{T} \mu=0, \mu \in\left(\mathscr{F}_{1}-\mathscr{F}_{2}\right)^{\circ} \Rightarrow \mu=0
$$

holds for every pair of faces $\mathscr{F}_{1}, \mathscr{F}_{2}$ with $\nabla \tilde{g}(\bar{y})(v) \in \mathscr{F}_{2} \subset \mathscr{F}_{1} \subset[\eta]^{\perp}$. By taking into account that $\left(\mathscr{F}_{1}-\mathscr{F}_{2}\right)^{\circ} \subset\left(\mathscr{F}_{2}-\mathscr{F}_{2}\right)^{\circ}$, the statement of the lemma follows.

This characterization of directional metric regularity can be considerably simplified.

Theorem 2.11 Let $\bar{y} \in \tilde{g}^{-1}(D)$ and $v \in \mathbb{R}^{m}$ be given and assume that $\Theta(\bar{y}, v) \neq \emptyset$. Then the following statements are equivalent:

1. There is some $(\bar{\lambda}, \bar{\eta}) \in \Theta(\bar{y}, v)$ such that the mapping $F$ given by (2.12) is metrically regular in direction $((v, \bar{\eta}),(0,0))$ at $((\bar{y}, \bar{\lambda}),(0,0))$.

2. The mapping $F$ given by (2.12) is metrically regular in direction $((v, \eta),(0,0))$ at $((\bar{y}, \lambda),(0,0))$ for every $(\lambda, \eta) \in \Theta(\bar{y}, v)$. 
3.

$$
\nabla \tilde{g}(\bar{y})^{T} \mu=0, \mu \in \operatorname{sp} N_{T_{D}(\tilde{g}(\bar{y}))}(\nabla \tilde{g}(\bar{y}) v) \Rightarrow \mu=0 .
$$

Proof Assume that the tangent cone $T_{D}(\tilde{g}(\bar{y}))$ has the representation (2.10) and consider any $(\lambda, \eta) \in \Theta(\bar{y}, v)$. Since $\eta \in N_{\mathscr{K}_{D}(\tilde{g}(\bar{y}), \lambda)}(\nabla \tilde{g}(\bar{y}) v)=\mathscr{K}_{D}(\tilde{g}(\bar{y}), \lambda)^{\circ} \cap[\nabla \tilde{g}(\bar{y}) v]^{\perp}$, $\nabla \tilde{g}(\bar{y}) v$ is contained in the face $\mathscr{K}_{D}(\tilde{g}(\bar{y}), \lambda)$ of $T_{D}(\tilde{g}(\bar{y}))$ and therefore

$$
\mathscr{J}(v):=\left\{i \in \bar{I} \mid a_{i}^{T} \nabla \tilde{g}(\bar{y}) v=0\right\} \supset J_{\lambda}:=J_{\mathscr{K}_{D}(\tilde{g}(\bar{y}), \lambda)},
$$

where $J_{\mathscr{K}_{D}(\tilde{g}(\bar{y}), \lambda)}$ is given by (2.11). Further, $\eta$ has the representation $\eta=\sum_{i \in I_{\eta}} \sigma_{i} a_{i}$ with $J_{\lambda} \subset I_{\eta} \subset \mathscr{J}(v)$ and $\sigma_{i}>0, i \in I_{\eta} \backslash J_{\lambda}$. Next consider any face $\mathscr{F}$ of the critical cone $\mathscr{K}_{D}(\tilde{g}(\bar{y}), \lambda)$ satisfying $\nabla \tilde{g}(\bar{y}) v \in \mathscr{F} \subset[\eta]^{\perp}$. Then $\mathscr{F}$ is again a face of $T_{D}(\tilde{g}(\bar{y}))$ and from $\nabla \tilde{g}(\bar{y}) v \in \mathscr{F} \subset[\eta]^{\perp}$ we deduce

$$
I_{\eta} \subset J_{\mathscr{F}} \subset \mathscr{J}(v)
$$

Thus

$$
\mathscr{F} \supset \mathscr{F}_{v}:=\left\{z \mid a_{i}^{T} z=0, i \in \mathscr{J}(v), a_{i}^{T} z \leq 0, i \in \bar{I} \backslash \mathscr{J}(v)\right\}
$$

and therefore $(\mathscr{F}-\mathscr{F})^{\circ} \subset\left(\mathscr{F}_{v}-\mathscr{F}_{v}\right)^{\circ}$. Since $\mathscr{F}_{v}$ is also a face of $\mathscr{K}_{D}(\tilde{g}(\bar{y}), \lambda)$ satisfying $\nabla \tilde{g}(\bar{y}) v \in \mathscr{F}_{v} \subset[\eta]^{\perp}$, by Lemma $2.10, F$ is metrically regular in direction $((v, \eta),(0,0))$ at $((\bar{y}, \lambda),(0,0))$ if and only if

$$
\nabla \tilde{g}(\bar{y})^{T} \mu=0, \mu \in\left(\mathscr{F}_{v}-\mathscr{F}_{v}\right)^{\circ} \Rightarrow \mu=0 .
$$

Since $\mathscr{F}_{v}$ depends neither on $\lambda$ nor on $\eta$, the equivalence between 1 . and 2 . is established. To show the equivalence of (2.14) with (2.13) just observe that $\mathscr{F}_{v}-\mathscr{F}_{v}=$ $\left\{z \quad \mid a_{i}^{T} z=0, i \in \mathscr{J}(v)\right\}$ implying $\left(\mathscr{F}_{v}-\mathscr{F}_{v}\right)^{\circ}=\left\{\sum_{i \in \mathscr{J}(v)} \sigma_{i} a_{i} \mid \sigma_{i} \in \mathbb{R}, i \in\right.$ $\mathscr{J}(v)\}$ and $N_{T_{D}(\tilde{g}(\bar{y}))}(\nabla \tilde{g}(\bar{y}) v)=\left\{\sum_{i \in \mathscr{J}(v)} \sigma_{i} a_{i} \mid \sigma_{i} \geq 0, i \in \mathscr{J}(v) \backslash \bar{J}\right\}$. Thus $\operatorname{sp} N_{T_{D}(\tilde{g}(\bar{y}))}(\nabla \tilde{g}(\bar{y}) v)=\left(\mathscr{F}_{v}-\mathscr{F}_{v}\right)^{\circ}$ and the proof is complete.

From the proof of Theorem 2.11 we also obtain the following corollary.

Corollary 2.12 Let $\bar{y} \in \tilde{g}^{-1}(D), \lambda \in N_{D}(\tilde{g}(\bar{y})), v \in \mathbb{R}^{m}$ and $\eta \in N_{\mathscr{K}_{D}(\tilde{g}(\bar{y}), \lambda)}(\nabla \tilde{g}(\bar{y}) v)$ be given. Then the union of all sets $(\mathscr{F}-\mathscr{F})^{\circ}$, where $\mathscr{F}$ is a face of the critical cone $\mathscr{K}_{D}(\tilde{g}(\bar{y}), \lambda)$ satisfying $\nabla \tilde{g}(\bar{y}) v \subset \mathscr{F} \subset[\eta]^{\perp}$, is exactly sp $N_{T_{D}(\tilde{g}(\bar{y}))}(\nabla \tilde{g}(\bar{y}) v)$.

Definition 2.13 Let $\bar{y} \in \tilde{g}^{-1}(D)$ and $v \in \mathbb{R}^{m}$ be given. We say that the system $\tilde{g}(\cdot) \in D$ is non-degenerate in direction $v$ at $\bar{y}$ if condition (2.13) is fulfilled. In case when $v=0$ we simply say that the system $\tilde{g}(\cdot) \in D$ is non-degenerate at $\bar{y}$.

Note that (2.13) is automatically fulfilled if $\nabla \tilde{g}(\bar{y}) v \notin T_{D}(\tilde{g}(\bar{y}))$. Further, if $\nabla \tilde{g}(\bar{y}) v \in$ $T_{D}(\tilde{g}(\bar{y}))$, then (2.13) is equivalent to

$$
\begin{aligned}
\mathbb{R}^{s} & \left.=\{0\}^{\perp}=\left(\operatorname{ker} \nabla \tilde{g}(\bar{y})^{T} \cap \operatorname{sp} N_{T_{D}(\tilde{g}(\bar{y}))}(\nabla \tilde{g}(\bar{y}) v)\right)\right)^{\perp}=\nabla \tilde{g}(\bar{y}) \mathbb{R}^{m}+\left(\operatorname{sp} N_{T_{D}(\tilde{g}(\bar{y}))}(\nabla \tilde{g}(\bar{y}) v)\right)^{\perp} \\
& =\nabla \tilde{g}(\bar{y}) \mathbb{R}^{m}+\left(N_{T_{D}(\tilde{g}(\bar{y}))}(\nabla \tilde{g}(\bar{y}) v)-N_{T_{D}(\tilde{g}(\bar{y}))}(\nabla \tilde{g}(\bar{y}) v)\right)^{\circ} \\
& =\nabla \tilde{g}(\bar{y}) \mathbb{R}^{m}+\left(N_{T_{D}(\tilde{g}(\bar{y}))}(\nabla \tilde{g}(\bar{y}) v)\right)^{\circ} \cap\left(-N_{T_{D}(\tilde{g}(\bar{y}))}(\nabla \tilde{g}(\bar{y}) v)\right)^{\circ}
\end{aligned}
$$

which in turn is equivalent to

$$
\nabla \tilde{g}(\bar{y}) \mathbb{R}^{m}+\operatorname{lin} T_{T_{D}(\tilde{g}(\bar{y}))}(\nabla \tilde{g}(\bar{y}) v)=\mathbb{R}^{s} .
$$

Clearly, for $v=0$ we obtain the standard definition of non-degeneracy from [3, Formula 4.172] and [32, Definition 2.1.]. 
We now state some properties of directional non-degeneracy.

Proposition 2.14 Let $\bar{y} \in \tilde{g}^{-1}(D)$ and $v \in \mathbb{R}^{n}$ such that the system $\tilde{g}(\cdot) \in D$ is nondegenerate in direction $v$ at $\bar{y}$. Then there is a directional neighborhood $\mathscr{V}$ of $v$ and a constant $\beta>0$ such that for all $y \in\left((\bar{y}+\mathscr{V}) \cap \tilde{g}^{-1}(D)\right), y \neq \bar{y}$, one has

$$
\left\|\nabla \tilde{g}(y)^{T} \mu\right\| \geq \beta\|\mu\| \forall \mu \in \operatorname{sp} N_{D}(\tilde{g}(y)) .
$$

In particular, for all $y \in\left((\bar{y}+\mathscr{V}) \cap \tilde{g}^{-1}(D)\right), y \neq \bar{y}$, the system $\tilde{g}(\cdot) \in D$ in non-degenerate at $y$.

Proof By contraposition. Assume on the contrary that there are sequences $t_{k} \downarrow 0, y_{k} \in$ $\tilde{g}^{-1}(D), \mu_{k} \in\left(\operatorname{sp} N_{D}\left(\tilde{g}\left(y_{k}\right)\right) \cap \mathscr{S}_{\mathbb{R}^{s}}\right.$ such that $\lim _{k \rightarrow \infty} \nabla \tilde{g}\left(y_{k}\right)^{T} \mu_{k}=0$ and $\lim _{k \rightarrow \infty}\left(y_{k}-\right.$ $\bar{y}) / t_{k} \rightarrow v$. Since for all $k$ sufficiently large we have

$$
\begin{aligned}
N_{D}\left(\tilde{g}\left(y_{k}\right)\right) & =\left(T_{D}(\tilde{g}(\bar{y}))^{\circ} \cap\left[\tilde{g}\left(y_{k}\right)-\tilde{g}(\bar{y})\right]^{\perp}=\left(T_{D}(\tilde{g}(\bar{y}))+\left[\frac{\tilde{g}\left(y_{k}\right)-\tilde{g}(\bar{y})}{t_{k}}\right]\right)^{\circ}\right. \\
& =\left(T_{T_{D}(\tilde{g}(\bar{y}))}\left(\frac{\tilde{g}\left(y_{k}\right)-\tilde{g}(\bar{y})}{t_{k}}\right)\right)^{\circ}=N_{T_{D}(\tilde{g}(\bar{y}))}\left(\frac{\tilde{g}\left(y_{k}\right)-\tilde{g}(\bar{y})}{t_{k}}\right) \\
& =N_{T_{D}(\tilde{g}(\bar{y}))}(\nabla \tilde{g}(\bar{y}) v) \cap\left[\frac{\tilde{g}\left(y_{k}\right)-\tilde{g}(\bar{y})}{t_{k}}-\nabla \tilde{g}(\bar{y}) v\right]^{\perp} \subset N_{T_{D}(\tilde{g}(\bar{y}))}(\nabla \tilde{g}(\bar{y}) v),
\end{aligned}
$$

it holds that $\mu_{k} \in \operatorname{sp} N_{T_{D}(\tilde{g}(\bar{y}))}(\nabla \tilde{g}(\bar{y}) v)$ and, by passing to some subsequence if necessary, we can assume that $\mu_{k}$ converges to some $\mu \in\left(\operatorname{sp} N_{T_{D}(\tilde{g}(\bar{y}))}(\nabla \tilde{g}(\bar{y}) v)\right) \cap \mathscr{S}_{\mathbb{R}^{s}}$. Obviously we also have $\nabla g(\bar{y})^{T} \mu=0$, a contradiction to the assumed directional non-degeneracy and (2.16) is proved. The additional statement concerning the non-degeneracy is an immediate consequence of (2.16).

It turns out that the directional non-degeneracy can be fulfilled in all non-zero directions even if the (standard) non-degeneracy is violated.

Example 2.15 Let $D=\mathbb{R}_{-}^{s}$ and assume $\tilde{g}(\bar{y})=0$. Given a direction $v$ satisfying $\nabla \tilde{g}(\bar{y}) v \leq 0$, we have $\operatorname{sp} N_{T_{D}(\tilde{g}(\bar{y}))}(\nabla \tilde{g}(\bar{y}) v)=\left\{\mu \in \mathbb{R}^{s} \mid \mu_{i}=0, i \notin \mathscr{J}(v)\right\}$, where $\mathscr{J}(v):=\left\{i \mid \nabla \tilde{g}_{i}(\bar{y}) v=0\right\}$. Thus, non-degeneracy in direction $v$ is equivalent to the linear independence of the gradients $\nabla \tilde{g}_{i}(\bar{y}), i \in \mathscr{J}(v)$ whereas non-degeneracy amounts to the so-called linear independence constraint qualification (LICQ), i.e., to the linear independence of all gradients $\nabla \tilde{g}_{i}(\bar{y}), i=1, \ldots, s$.

Consider the system

$y_{1}-y_{4} \leq 0,-y_{1}-y_{4} \leq 0, y_{2}-y_{4} \leq 0,-y_{2}-y_{4} \leq 0, y_{3}+y_{1}^{2}-y_{4} \leq 0,-y_{3}-y_{4} \leq 0$.

Obviously LICQ is violated at $\bar{y}=0$. However, it is not difficult to verify that the system is non-degenerate in every direction $v \neq 0$.

Further note that in this example also the so-called constant rank constraint qualification is violated at $\bar{y}$.

\section{Stability Properties Through Generalized Differentiation}

Throughout this section we consider the solution mapping $S$ given by (1.2). Given some reference point $(\bar{p}, \bar{x}) \in \operatorname{gph} S$, we will provide point-based sufficient conditions for the 
isolated calmness property, the Aubin property and the Aubin property relative to some set $P \subset \mathbb{R}^{l}$, respectively, in terms of generalized derivatives of the mapping $M$.

We start with the Levy-Rockafellar characterization of isolated calmness [27], who showed that

$$
S \text { is isolated calm at }(\bar{p}, \bar{x}) \in \operatorname{gph} S \Leftrightarrow D S(\bar{p}, \bar{x})(0)=\{0\} \text {. }
$$

Theorem 3.1 Assume that $M$ has locally closed graph around the reference point $(\bar{p}, \bar{x}, 0) \in \operatorname{gph} M$. If

$$
0 \in \operatorname{DM}(\bar{p}, \bar{x}, 0)(0, u) \Rightarrow u=0,
$$

then $S$ has the isolated calmness property at $(\bar{p}, \bar{x})$. Conversely, if there is some $u \neq 0$ such that $0 \in \operatorname{DM}(\bar{p}, \bar{x}, 0)(0, u)$ and $M$ is metrically subregular in direction $(0, u)$ then $S$ is not isolatedly calm at $(\bar{p}, \bar{x})$.

Proof Note that the closedness of gph $M$ readily implies that gph $S=M^{-1}(0)$ is locally closed around $(\bar{p}, \bar{x})$. The sufficiency of (3.18) for the isolated calmness property of $S$ is due to (3.17) together with the inclusion

$$
D S(\bar{p}, \bar{x})(0) \subset\{u \mid 0 \in D M(\bar{p}, \bar{x}, 0)(0, u)\}
$$

following from the definition of the graphical derivative, see also [27, Theorem 3.1]. In order to show the second statement, consider $u \neq 0$ verifying $0 \in D M(\bar{p}, \bar{x}, 0)(0, u)$ and assume that $M$ is metrically subregular in direction $(0, u)$ at $(\bar{p}, \bar{x}, 0)$. By [16, Proposition 4.1] we obtain $(0, u) \in T_{M^{-1}(0)}(\bar{p}, \bar{x})=T_{\operatorname{gph} S}(\bar{p}, \bar{x})$ and consequently $u \in D S(\bar{p}, \bar{x})(0)$. Thus mapping $S$ is not isolatedly calm at $(\bar{p}, \bar{x})$ by (3.17).

Since metric subregularity of $M$ implies metric subregularity in any direction, we obtain the following corollary.

Corollary 3.2 Assume that $M$ has locally closed graph around and is metrically subregular at $(\bar{p}, \bar{x}, 0) \in \operatorname{gph} M$. Then $S$ is isolatedly calm at $(\bar{p}, \bar{x})$ if and only if (3.18) holds.

A sufficient condition for the Aubin property of $S$ around $(\bar{p}, \bar{x})$ is constituted by the following theorem.

Theorem 3.3 ([16, Theorem 4.4]) Assume that $M$ has locally closed graph around the reference point $(\bar{p}, \bar{x}, 0) \in \operatorname{gph} M$ and assume that

$$
\left\{u \in \mathbb{R}^{n} \mid 0 \in \operatorname{DM}(\bar{p}, \bar{x}, 0)(q, u)\right\} \neq \emptyset \text { for all } q \in \mathbb{R}^{l} ;
$$

(ii) $M$ is metrically subregular at $(\bar{p}, \bar{x}, 0)$;

(iii) For every nonzero $(q, u) \in \mathbb{R}^{l} \times \mathbb{R}^{n}$ verifying $0 \in D M(\bar{p}, \bar{x}, 0)(q, u)$ one has the implication

$$
\left(q^{*}, 0\right) \in D^{*} M((\bar{p}, \bar{x}, 0) ;(q, u, 0))\left(v^{*}\right) \Rightarrow q^{*}=0 .
$$

Then $S$ has the Aubin property around $(\bar{p}, \bar{x})$ and for any $q \in \mathbb{R}^{l}$

$$
D S(\bar{p}, \bar{x})(q)=\{u \mid 0 \in \operatorname{DM}(\bar{p}, \bar{x}, 0)(q, u)\} .
$$

The above assertions remain true provided assumptions (ii), (iii) are replaced by 
(iv) For every nonzero $(q, u) \in \mathbb{R}^{l} \times \mathbb{R}^{n}$ verifying $0 \in D M(\bar{p}, \bar{x}, 0)(q, u)$ one has the implication

$$
\left(q^{*}, 0\right) \in D^{*} M((\bar{p}, \bar{x}, 0) ;(q, u, 0))\left(v^{*}\right) \Rightarrow\left\{\begin{array}{l}
q^{*}=0 \\
v^{*}=0
\end{array}\right.
$$

Sufficient conditions for the Aubin property of $S$ relative to some set $P$ are based on the following statement.

Proposition 3.4 Let $(\bar{p}, \bar{x}, 0) \in \operatorname{gph} M$ and consider a subset $P \subset \mathbb{R}^{l}$ containing $\bar{p}$, equipped with the induced norm topology of $\mathbb{R}^{l}$. If the system $0 \in M(p, x)$, rewritten as

$$
h(p, x) \in \operatorname{gph} M
$$

with $h(p, x):=(p, x, 0)$, enjoys the Robinson stability property at $(\bar{p}, \bar{x})$, then $S$ has the Aubin property relative to $P$ around $(\bar{p}, \bar{x})$.

Proof Obviously $S$ is also the solution mapping of the inclusion $(p, x, 0) \in \operatorname{gph} M$. By the definition of the Robinson stability together with the assumption on the topology of $P$, there are neighborhoods $Q$ of $\bar{p}$ in $\mathbb{R}^{l}, U$ of $\bar{x}$ and a constant $\kappa \geq 0$ such that

$$
\operatorname{dist}(x, S(p)) \leq \kappa \operatorname{dist}((p, x, 0), \operatorname{gph} M) \forall(p, x) \in(Q \cap P) \times U .
$$

Next consider $p, p^{\prime} \in Q \cap P$ and $x \in S(p) \cap U$. Then

$$
\operatorname{dist}\left(x, S\left(p^{\prime}\right)\right) \leq \kappa \operatorname{dist}\left(\left(p^{\prime}, x, 0\right), \operatorname{gph} M\right) \leq \kappa\left(\operatorname{dist}((p, x, 0), \operatorname{gph} M)+\left\|p-p^{\prime}\right\|\right)=\kappa\left\|p-p^{\prime}\right\|
$$

and thus $x \in S\left(p^{\prime}\right)+(\kappa+1)\left\|p-p^{\prime}\right\| \mathscr{B}_{\mathbb{R}^{n}}$. It follows that $S(p) \cap U \subset S\left(p^{\prime}\right)+(\kappa+$ 1) $\left\|p-p^{\prime}\right\| \mathscr{B}_{\mathbb{R}^{n}}$ showing the Aubin property of $S$ relative to $P$.

Theorem 3.5 Assume that $M$ has a locally closed graph around the reference point $(\bar{p}, \bar{x}, 0) \in \operatorname{gph} M$ and consider a closed set $P \subset \mathbb{R}^{l}$ containing $\bar{p}$. Further assume that

(i) for every $q \in T_{P}(\bar{p})$ and every sequence $t_{k} \downarrow 0$ there exists some $u \in \mathbb{R}^{n}$ satisfying

$$
\liminf _{k \rightarrow \infty} \operatorname{dist}\left(\left(\bar{p}+t_{k} q, \bar{x}+t_{k} u, 0\right), \operatorname{gph} M\right) / t_{k}=0
$$

(ii) For every nonzero $(q, u) \in T_{P}(\bar{p}) \times \mathbb{R}^{n}$ verifying $0 \in D M(\bar{p}, \bar{x}, 0)(q, u)$ one has the implication

$$
\left(q^{*}, 0\right) \in D^{*} M((\bar{p}, \bar{x}, 0) ;(q, u, 0))\left(v^{*}\right) \Rightarrow\left\{\begin{array}{l}
q^{*}=0 \\
v^{*}=0 .
\end{array}\right.
$$

Then $S$ has the Aubin property relative to $P$ around $(\bar{p}, \bar{x})$ and for any $q \in T_{P}(\bar{p})$

$$
D S(\bar{p}, \bar{x})(q)=\{u \mid 0 \in D M(\bar{p}, \bar{x}, 0)(q, u)\} .
$$

Proof First, we apply [14, Corollary 3.6] to show the Robinson stability property of the system (3.19) at $(\bar{p}, \bar{x})$. By taking $\zeta(p)=\|p-\bar{p}\|$ we obtain that the image derivative $\operatorname{Im}_{\zeta} D_{p} h(\bar{p}, \bar{x})$ defined in [14] as the closed cone generated by 0 and those $v \in \mathbb{R}^{l} \times \mathbb{R}^{n} \times \mathbb{R}^{m}$ for which there is a sequence $\left\{p_{k}\right\} \subset P$ with

$$
\begin{array}{r}
0<\left\|h\left(p_{k}, \bar{x}\right)-h(\bar{p}, \bar{x})\right\|<k^{-1},\left\|\nabla_{x} h\left(p_{k}, \bar{x}\right)-\nabla_{x} h(\bar{p}, \bar{x})\right\|<k^{-1},\left|\zeta\left(p_{k}\right)-\zeta(\bar{p})\right|<k^{-1}, \\
v=\lim _{k \rightarrow \infty} \frac{h\left(p_{k}, \bar{x}\right)-h(\bar{p}, \bar{x})}{\left\|h\left(p_{k}, \bar{x}\right)-h(\bar{p}, \bar{x})\right\|}=\lim _{k \rightarrow \infty} \frac{\left(p_{k}-\bar{p}, 0,0\right)}{\left\|p_{k}-\bar{p}\right\|},
\end{array}
$$


is exactly the set $\left\{(q, 0,0) \mid q \in T_{P}(\bar{p})\right\}$. Further for every $u \in \mathbb{R}^{n}$ we have $\nabla_{x} h(\bar{p}, \bar{x}) u=$ $(0, u, 0)$ and thus [14, Condition 3.10] is fulfilled by (3.20). Next we have to verify that for every pair $(0,0) \neq(q, u) \in T_{P}(\bar{p}) \times \mathbb{R}^{n}$ satisfying $(q, u, 0) \in T_{\operatorname{gph} M}(\bar{p}, \bar{x}, 0)$ the implication

$$
\lambda \in N_{\mathrm{gph} M}((\bar{p}, \bar{x}, 0),(q, u, 0)), \nabla_{x} h(\bar{p}, \bar{x})^{T} \lambda=0 \Rightarrow \lambda=0
$$

is fulfilled. Setting $\lambda:=\left(q^{*}, u^{*},-v^{*}\right)$ this amounts to

$$
\left(q^{*}, u^{*}\right) \in D^{*} M((\bar{p}, \bar{x}, 0) ;(q, u, 0))\left(v^{*}\right), u^{*}=0 \Rightarrow\left(q^{*}, u^{*},-v^{*}\right)=(0,0,0),
$$

which is obviously equivalent to (3.21). By taking into account that the condition $(q, u, 0) \in T_{\operatorname{gph} M}(\bar{p}, \bar{x}, 0)$ is the same as requiring $0 \in D M(\bar{p}, \bar{x}, 0)(q, u)$, all assumption of [14, Corollary 3.6] are fulfilled and the claimed Robinson stability property of the system (3.19) at $(\bar{p}, \bar{x})$ follows. By virtue of Proposition 3.4 this implies the Aubin property of $S$ relative to $P$ around $(\bar{p}, \bar{x})$. There remains to show (3.22). Since $\{u \mid$ $0 \in D M(\bar{p}, \bar{x}, 0)(q, u)\} \supset D S(\bar{p}, \bar{x})(q)$ always holds by [27, Theorem 3.1], we only have to show $\{u \mid 0 \in D M(\bar{p}, \bar{x}, 0)(q, u)\} \subset D S(\bar{p}, \bar{x})(q)$. Consider $u$ satisfying $0 \in D M(\bar{p}, \bar{x}, 0)(q, u)$ for some $q \in T_{P}(\bar{p})$. By Theorem 2.6, condition (3.21) implies that $M$ is metrically subregular in direction $(q, u)$ at $(\bar{p}, \bar{x}, 0)$ and hence we can invoke [16, Proposition 4.1] to obtain $(q, u) \in T_{M^{-1}(0)}(\bar{p}, \bar{x})=T_{\mathrm{gph} S}(\bar{p}, \bar{x})$ and consequently $u \in D S(\bar{p}, \bar{x})(q)$. Thus $\{u \mid 0 \in D M(\bar{p}, \bar{x}, 0)(q, u)\} \subset D S(\bar{p}, \bar{x})(q)$ and the proof of the theorem is complete.

Remark 3.6 By virtue of Theorem 2.6, the mapping $M$ is metrically regular in direction $(q, u, 0)$ at $(\bar{p}, \bar{x}, 0)$ if and only if

$$
\left.(0,0) \in D^{*} M(\bar{p}, \bar{x}, 0) ;(q, u, 0)\right)\left(v^{*}\right) \Rightarrow v^{*}=0 .
$$

Thus, condition (ii) of Theorem 3.5 implies that for every nonzero $(q, u) \in T_{P}(\bar{p}) \times \mathbb{R}^{n}$ verifying $0 \in D M(\bar{p}, \bar{x}, 0)(q, u)$ the mapping $M$ is metrically regular in direction $(q, u, 0)$.

Remark 3.7 Let us briefly compare the above theorems. Theorem 3.3 was directly obtained in this form in [16]. As a result, it offers the more refined result, based on assumptions (ii) and (iii), as well as the simplified version using condition (iv) instead. On the other hand, Theorem 3.5 is based on the result for the Robinson stability property from [14], which enables us to consider perturbations restricted to set $P$. Compared to Theorem 3.3, it is only available under the stronger assumption (3.21). Assumption (i) of Theorem 3.5 is fulfilled in particular if for every $q \in T_{P}(\bar{p})$ there is some $u \in \mathbb{R}^{n}$ satisfying $0 \in D M(\bar{p}, \bar{x}, 0)(q, u)$ and the tangent $(q, u, 0)$ to gph $M$ is derivable. We see that in this case Theorem 3.5 is a generalization of (the simplified form of) Theorem 3.3.

\section{Graphical Derivative of the Normal Cone Mapping}

This section deals with computation of the graphical derivative of $M$ given by (1.3). Throughout the rest of the paper we assume that we are given a reference solution $(\bar{p}, \bar{x})$ of (1.3) fulfilling the following assumption.

Assumption 1 There is some $\kappa>0$ such that for all $(p, x, z)$ belonging to a neighborhood of $(\bar{p}, \bar{x}, \bar{x})$ the inequality

$$
\operatorname{dist}(z, \Gamma(p, x)) \leq \kappa \operatorname{dist}(g(p, x, z), D)
$$


holds.

Note that by Theorem 2.8 Assumption 1 is fulfilled, e.g., in the case when

$$
\nabla_{3} g(\bar{p}, \bar{x}, \bar{x})^{T} \mu=0, \mu \in N_{D}(g(\bar{p}, \bar{x}, \bar{x})) \Rightarrow \mu=0
$$

which is equivalent to Robinson's constraint qualification

$$
\nabla_{3} g(\bar{p}, \bar{x}, \bar{x}) \mathbb{R}^{n}+T_{D}(g(\bar{p}, \bar{x}, \bar{x}))=\mathbb{R}^{s} .
$$

As a consequence of Assumption 1 we obtain that for all $(p, x, z) \in \operatorname{gph} \Gamma$ sufficiently close to $(\bar{p}, \bar{x}, \bar{x})$ the mapping $g(p, x, \cdot)-D$ is metrically subregular at $(z, 0)$ with modulus $\kappa$ and therefore, by virtue of [21, Theorem 5]

$$
\widehat{N}_{\Gamma(p, x)}(z)=N_{\Gamma(p, x)}(z)=\nabla_{3} g(p, x, z)^{T} N_{D}(g(p, x, z)) .
$$

Moreover, for every $z^{*} \in \widehat{N}_{\Gamma(p, x)}(z)$ there is a multiplier $\lambda \in N_{D}(g(p, x, z))$ with

$$
z^{*}=\nabla_{3} g(p, x, z)^{T} \lambda,\|\lambda\| \leq \kappa\left\|z^{*}\right\|,
$$

cf. [14, Lemma 2.1]. Finally, since $\operatorname{gph} \Gamma=\{(p, x, z) \mid g(p, x, z) \in D\}$ and $\operatorname{dist}((p, x, z), \operatorname{gph} \Gamma) \leq \operatorname{dist}(z, \Gamma(p, x))$, we conclude that the mapping $g(\cdot)-D$ is metrically subregular at $((p, x, z), 0)$ for every $(p, x, z) \in \operatorname{gph} \Gamma$ sufficiently close to $(\bar{p}, \bar{x}, \bar{x})$. Therefore

$$
\begin{gathered}
T_{\mathrm{gph} \Gamma}(p, x, z)=\left\{(q, u, w) \in \mathbb{R}^{l} \times \mathbb{R}^{n} \times \mathbb{R}^{n} \mid \nabla g(p, x, z)(q, u, w) \in T_{D}(g(p, x, z))\right\}, \\
\widehat{N}_{\mathrm{gph} \Gamma}(p, x, z)=\nabla g(p, x, z)^{T} N_{D}(g(p, x, z)) .
\end{gathered}
$$

In order to unburden the notation we introduce the mappings

$$
b(p, x):=\nabla_{3} g(p, x, x), \quad \tilde{g}(p, x):=g(p, x, x)
$$

and denote the set-valued part of $M(p, x)$ as $G(p, x):=\widehat{N}_{\Gamma(p, x)}(x)$. For $(p, x)$ close to $(\bar{p}, \bar{x})$ one has

$$
G(p, x)=b(p, x)^{T} N_{D}(\tilde{g}(p, x)) .
$$

The graphical derivative of $G$ is closely related with the graphical derivative of the mapping $\Psi: \mathbb{R}^{l} \times \mathbb{R}^{n} \times \mathbb{R}^{n} \rightrightarrows \mathbb{R}^{n}$ given by

$$
\Psi(p, x, z):=\widehat{N}_{\Gamma(p, x)}(z) .
$$

In order to give a formula for the graphical derivative of $\psi$ we employ the following notation. Given any $y:=(p, x, z) \in \operatorname{gph} \Gamma$ and any $y^{*}=\left(p^{*}, x^{*}, z^{*}\right) \in \widehat{N}_{\mathrm{gph}} \Gamma(y)$, we denote by

$$
\Lambda\left(y, y^{*}\right):=\left\{\lambda \in N_{D}(g(y)) \mid \nabla g(y)^{T} \lambda=y^{*}\right\}
$$

the corresponding set of multipliers and for any $v=(q, u, w) \in \mathbb{R}^{l} \times \mathbb{R}^{n} \times \mathbb{R}^{n}$ by

$$
\Lambda\left(y, y^{*} ; v\right):=\arg \max \left\{v^{T} \nabla^{2}\left\langle\lambda^{T} g\right\rangle(y) v \mid \lambda \in \Lambda\left(y, y^{*}\right)\right\}
$$

the directional set of multipliers. Further, for any $y^{*}=\left(p^{*}, x^{*}, z^{*}\right) \in \mathbb{R}^{l} \times \mathbb{R}^{n} \times \mathbb{R}^{n}$ we denote by $\pi_{3}\left(y^{*}\right)$ the canonical projection of $y^{*}$ on its third component, i.e., $\pi_{3}\left(y^{*}\right)=z^{*}$. The next statement is a counterpart of $[18$, Proposition 1] and uses a substantially weaker assumption. 
Proposition 4.1 Assume that Assumption 1 is fulfilled. Then for all $y:=(p, x, z) \in \operatorname{gph} \Gamma$ sufficiently close to $(\bar{p}, \bar{x}, \bar{x})$, all $z^{*} \in \Psi(y)$ and all $v:=(q, u, w) \in \mathbb{R}^{l} \times \mathbb{R}^{n} \times \mathbb{R}^{n}$ we have

$$
\begin{aligned}
& D \Psi\left(y, z^{*}\right)(v) \\
& =\left\{\nabla\left(\nabla_{3} g(\cdot)^{T} \lambda\right)(y) v+\pi_{3}\left(N_{\mathscr{K}_{\mathrm{gph} \Gamma}\left(y, y^{*}\right)}(v)\right) \mid y^{*} \in N_{T_{\mathrm{gph} \Gamma}(y)}(v), \pi_{3}\left(y^{*}\right)=z^{*}, \lambda \in \Lambda\left(y, y^{*} ; v\right)\right\} \\
& =\left\{\nabla\left(\nabla_{3} g(\cdot)^{T} \lambda\right)(y) v+\nabla_{3} g(y)^{T} N_{\mathscr{K}_{D}(g(y), \lambda)}(\nabla g(y) v) \mid \lambda \in \Lambda\left(y, \nabla g(y)^{T} \mu ; v\right),\right. \\
& \left.\nabla_{3} g(y)^{T} \mu=z^{*}, \mu \in N_{D}(g(y)), \mu^{T} \nabla g(y) v=0\right\} .
\end{aligned}
$$

Proof The first equality is an immediate consequence of [15, Theorem 5.3]. By $y^{*} \in$

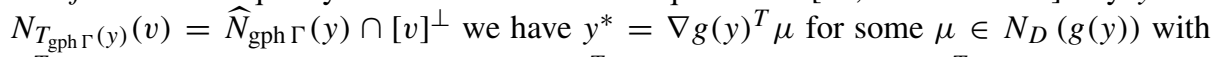
$\mu^{T} \nabla g(y) v=0$ and due to $\lambda \in \Lambda\left(y, \nabla g(y)^{T} \mu ; v\right)$ we also have $\nabla g(y)^{T} \lambda=y^{*}$. Since

$\mathscr{K}_{\mathrm{gph} \Gamma}\left(y, y^{*}\right)=\mathscr{K}_{\mathrm{gph} \Gamma}\left(y, \nabla g(y)^{T} \lambda\right)=\left\{v \mid \nabla g(y) v \in T_{D}(g(y)), \lambda^{T} \nabla g(y) v=0\right\}=\nabla g(y)^{-1} \mathscr{K}_{D}(g(y), \lambda)$,

we obtain $\mathscr{K}_{\text {gph } \Gamma}\left(y, y^{*}\right)^{\circ}=\nabla g(y)^{T} \mathscr{K}_{D}(g(y), \lambda)^{\circ}$ by [33, Corollary 16.3.2] and by taking into account that the set $\nabla g(y)^{T} \mathscr{K}_{D}(g(y), \lambda)^{\circ}$ is a convex polyhedral cone and therefore closed. Thus

$$
\begin{aligned}
N_{\mathscr{K}_{\mathrm{gph} \Gamma}\left(y, y^{*}\right)}(v)=\mathscr{K}_{\mathrm{gph} \Gamma}\left(y, y^{*}\right)^{\circ} \cap[v]^{\perp} & =\left\{\nabla g(y)^{T} \eta \mid \eta \in \mathscr{K}_{D}(g(y), \lambda)^{\circ}, v^{T} \nabla g(y)^{T} \eta=0\right\} \\
& =\nabla g(y)^{T} N_{\mathscr{K}_{D}(g(y), \lambda)}(\nabla g(y) v)
\end{aligned}
$$

showing $\pi_{3}\left(N_{\mathscr{K}_{\mathrm{gph} \Gamma}\left(y, y^{*}\right)}(v)\right)=\nabla_{3} g(y)^{T} N_{\mathscr{K}_{D}(g(y), \lambda)}(\nabla g(y) v)$ and the proof is complete.

$$
\begin{aligned}
& \Xi\left((p, x), x^{*}\right):=\left\{\mu \in N_{D}(\tilde{g}(p, x)) \mid b(p, x)^{T} \mu=x^{*}\right\}, \\
& \Xi\left((p, x), x^{*} ;(q, u)\right):=\left\{\mu \in \Xi\left((p, x), x^{*}\right) \mid \nabla \tilde{g}(p, x)(q, u) \in \mathscr{K}_{D}(\tilde{g}(p, x), \mu)\right\}, \\
& \tilde{\Lambda}\left((p, x), x^{*} ;(q, u)\right):=\left\{\lambda \in \Lambda\left((p, x, x), \nabla g(p, x, x)^{T} \mu ;(q, u, u)\right) \mid \mu \in \Xi\left((p, x), x^{*} ;(q, u)\right)\right\}
\end{aligned}
$$

defined for $\left(p, x, x^{*}\right) \in \operatorname{gph} G$ and directions $(q, u) \in \mathbb{R}^{l} \times \mathbb{R}^{n}$. The next theorem generalizes [18, Theorem 2]. In particular, it provides a useful additional derivability result.

Theorem 4.2 Assume that Assumption 1 is fulfilled. Then for all $(p, x) \in \operatorname{dom} G$ sufficiently close to $(\bar{p}, \bar{x})$, all $x^{*} \in G(p, x)$ and all $(q, u) \in \mathbb{R}^{l} \times \mathbb{R}^{n}$ we have

$$
\begin{aligned}
& D G\left((p, x), x^{*}\right)(q, u) \subset D \Psi\left((p, x, x), x^{*}\right)(q, u, u) \\
&=\left\{\nabla\left(b(\cdot)^{T} \lambda\right)(p, x)(q, u)+b(p, x)^{T} N_{\mathscr{K}_{D}(\tilde{g}(p, x), \lambda)}(\nabla \tilde{g}(p, x)(q, u))\right. \text { (4.25) } \\
&\left.\qquad \lambda \in \tilde{\Lambda}\left((p, x), x^{*} ;(q, u)\right)\right\} .
\end{aligned}
$$

On the other hand, given $(q, u) \in \mathbb{R}^{l} \times \mathbb{R}^{n}, \lambda \in \tilde{\Lambda}\left((p, x), x^{*} ;(q, u)\right)$ and $\eta \in$ $N_{\mathscr{K}_{D}(\tilde{g}(p, x), \lambda)}(\nabla \tilde{g}(p, x)(q, u))$, assume that the mapping $F: \mathbb{R}^{l} \times \mathbb{R}^{n} \times \mathbb{R}^{s} \rightrightarrows \mathbb{R}^{s} \times \mathbb{R}^{s}$ given by

$$
F\left(p^{\prime}, x^{\prime}, \mu\right):=\left(\tilde{g}\left(p^{\prime}, x^{\prime}\right), \mu\right)-\operatorname{gph} N_{D}
$$

is metrically subregular in direction $(q, u, \eta)$ at $((p, x, \lambda),(0,0))$. Then we have

$$
\nabla\left(b(\cdot)^{T} \lambda\right)(p, x)(q, u)+b(p, x)^{T} \eta \in D G\left((p, x), x^{*}\right)(q, u)
$$

and the tangent $\left(q, u, \nabla\left(b(\cdot)^{T} \lambda\right)(p, x)(q, u)+b(p, x)^{T} \eta\right)$ to gph $G$ is derivable. 
Proof The inclusion (4.25) follows immediately from the definition of the graphical derivative, whereas (4.26) is a consequence of Proposition 4.1. Consider now $(q, u) \in \mathbb{R}^{l} \times \mathbb{R}^{n}$, $\lambda \in \tilde{\Lambda}\left((p, x), x^{*} ;(q, u)\right)$ and $\eta \in N_{\mathscr{K}_{D}(\tilde{g}(p, x), \lambda)}(\nabla \tilde{g}(p, x)(q, u))$ such that the mapping (4.27) is directionally metrically subregular. We conclude that

$$
(\nabla \tilde{g}(p, x)(q, u), \eta) \in \operatorname{gph} N_{\mathscr{K}_{D}(\tilde{g}(p, x), \lambda)}=T_{\operatorname{gph} N_{D}}(\tilde{g}(p, x), \lambda)
$$

and thus

$$
(\tilde{g}(p, x), \lambda)+t(\nabla \tilde{g}(p, x)(q, u), \eta) \in \operatorname{gph} N_{D}
$$

for all $t \geq 0$ sufficiently small, because gph $N_{D}$ is a polyhedral set.

Consequently we have

$$
\operatorname{dist}\left((\tilde{g}(p+t q, x+t u), \lambda+t \eta), \operatorname{gph} N_{D}\right)=o(t)
$$

and by the assumed directional metric subregularity of $F$ we can find for every $t>0$ some $\left(q_{t}, u_{t}, \eta_{t}\right)$ with $\lim _{t_{\downarrow} 0}\left(q_{t}, u_{t}, \eta_{t}\right)=(q, u, \eta)$ and $0 \in F\left(p+t q_{t}, x+t u_{t}, \lambda+t \eta_{t}\right)$ implying

$$
b\left(p+t q_{t}, x+t u_{t}\right)^{T}\left(\lambda+t \eta_{t}\right) \in G\left(p+t q_{t}, x+t u_{t}\right) .
$$

On the other hand, by Taylor expansion we obtain

$$
\begin{aligned}
b\left(p+t q_{t}, x+t u_{t}\right)^{T}\left(\lambda+t \eta_{t}\right) & =b(p, x)^{T} \lambda+t\left(\nabla\left(b(\cdot)^{T} \lambda\right)(p, x)(q, u)+b(p, x)^{T} \eta\right)+o(t) \\
& =x^{*}+t\left(\nabla\left(b(\cdot)^{T} \lambda\right)(p, x)(q, u)+b(p, x)^{T} \eta\right)+o(t)
\end{aligned}
$$

showing (4.28) and the derivability of the tangent $\left(q, u, \nabla\left(b(\cdot)^{T} \lambda\right)(p, x)(q, u)+b(p, x)^{T} \eta\right)$.

Theorem 4.3 Assume that Assumption 1 is fulfilled and assume that we are given $(p, x) \in \tilde{g}^{-1}(D)$ sufficiently close to $(\bar{p}, \bar{x}), x^{*} \in G(p, x)$ and $(q, u) \in \mathbb{R}^{l} \times \mathbb{R}^{n}$ with $\Xi\left((p, x), x^{*} ;(q, u)\right) \neq \emptyset$.

(i) Assume that for every $\lambda \in \tilde{\Lambda}\left((p, x), x^{*} ;(q, u)\right)$ and every $\eta \in$ $N_{\mathscr{K}_{D}(\tilde{g}(p, x), \lambda)}(\nabla \tilde{g}(p, x)(q, u))$ the mapping $F$ given by (4.27) is metrically subregular in direction $((q, u), \eta)$. Then

$$
D G\left((p, x), x^{*}\right)(q, u)=D \Psi\left((p, x, x), x^{*}\right)(q, u, u)
$$

and all tangents $\left(q, u, v^{*}\right) \in T_{\mathrm{gph} G}\left((p, x), x^{*}\right)$ are derivable.

(ii) If the system $\tilde{g}(\cdot) \in D$ is non-degenerate in direction $(q, u)$ at $(p, x)$ then (4.29) holds, all tangents $\left(q, u, v^{*}\right) \in T_{\operatorname{gph} G}\left((p, x), x^{*}\right)$ are derivable and for all $\mu \in$ $\Xi\left((p, x), x^{*} ;(q, u)\right)$ the set $\Lambda\left((p, x, x), \nabla g(p, x, x)^{T} \mu ;(q, u, u)\right)$ is the singleton $\{\mu\}$. Moreover, there is a directional neighborhood $\mathscr{V}$ of $(q, u)$ such that for all $\left(p^{\prime}, x^{\prime}\right) \in((p, x)+\mathscr{V}) \cap \tilde{g}^{-1}(D),\left(p^{\prime}, x^{\prime}\right) \neq(p, x)$, the system $\tilde{g}(\cdot)-D$ is non-degenerate at $\left(p^{\prime}, x^{\prime}\right)$ and for every $x^{* \prime} \in G\left(p^{\prime}, x^{\prime}\right)$ we have

$$
D G\left(\left(p^{\prime}, x^{\prime}\right), x^{* \prime}\right)\left(q^{\prime}, u^{\prime}\right)=D \Psi\left(\left(p^{\prime}, x^{\prime}, x^{\prime}\right), x^{* \prime}\right)\left(q^{\prime}, u^{\prime}, u^{\prime}\right) \forall\left(q^{\prime}, u^{\prime}\right) \in \mathbb{R}^{l} \times \mathbb{R}^{n} .
$$

Proof (i) follows immediately from Theorem 4.2. In order to show the second statement, note that by Theorem 2.11 the directional non-degeneracy of $\tilde{g}(\cdot) \in D$ in direction $(q, u)$ implies the assumptions of $(i)$ and therefore (4.29) follows. In order to show $\Lambda\left((p, x, x), \nabla g(p, x, x)^{T} \mu,(q, u, u)\right)=\{\mu\} \forall \mu \in \Xi\left((p, x), x^{*} ;(q, u)\right)$, fix $\mu \in$ $\Xi\left((p, x), x^{*} ;(q, u)\right)$ and consider the feasible set

$$
T:=\Lambda\left((p, x, x), \nabla g(p, x, x)^{T} \mu\right)=\left\{\zeta \in N_{D}(\tilde{g}(p, x)) \mid \nabla g(p, x, x)^{T} \zeta=\nabla g(p, x, x)^{T} \mu\right\}
$$


of the linear program defining $\Lambda\left((p, x, x), \nabla g(p, x, x)^{T} \mu,(q, u, u)\right)$. We claim that $T=\{\mu\}$. Indeed, $\mu \in T$ and consider any element $\zeta \in T$. Since $\nabla \tilde{g}(p, x)=\left(\nabla_{1} g(p, x, x)\right.$, $\left.\nabla_{2} g(p, x, x)+\nabla_{3} g(p, x, x)\right)$, we readily obtain $\nabla \tilde{g}(p, x)^{T} \zeta=\nabla \tilde{g}(p, x)^{T} \mu$. By definition of $\Xi\left((p, x), x^{*} ;(q, u)\right)$ we also have $\zeta^{T} \nabla \tilde{g}(p, x)(q, u)=\mu^{T} \nabla \tilde{g}(p, x)(q, u)=0$ implying $\zeta, \mu \in N_{T_{D}(\tilde{g}(p, x))}(\nabla \tilde{g}(p, x)(q, u))$. Thus $\nabla \tilde{g}(p, x)^{T}(\zeta-\mu)=0, \zeta-\mu \epsilon$ $\operatorname{sp} N_{T_{D}(\tilde{g}(p, x))}(\nabla \tilde{g}(p, x)(q, u))$ and we deduce $\zeta-\mu=0$ from the assumed directional non-degeneracy showing $T=\{\mu\}$. Now $\Lambda\left((p, x, x), \nabla g(p, x, x)^{T} \mu,(q, u, u)\right)=\{\mu\}$ follows immediately from the definition. The last part of (ii) is implied by Proposition 2.14 taking into account that non-degeneracy of $\tilde{g}(\cdot)-D$ at $\left(p^{\prime}, x^{\prime}\right)$ implies non-degeneracy in any direction and by Remark 4.4 below.

Remark 4.4 Note that in case when $\Xi\left((p, x), x^{*} ;(q, u)\right)=\varnothing$ we have $D \Psi\left((p, x, x), x^{*}\right)(q, u, u)=\emptyset$ and thus the equality (4.29) automatically holds by virtue of (4.25). In particular we have $D G\left((p, x), x^{*}\right)(q, u)=D \Psi\left((p, x, x), x^{*}\right)(q, u, u)=\varnothing$ for all directions $(q, u)$ with $\nabla \tilde{g}(p, x)(q, u)=\nabla g(p, x, x)(q, u, u) \notin T_{D}(\tilde{g}(p, x))$.

Observe that Theorem (4.3) (ii) extends the results stated in [18, Theorem 2 and Proposition 2].

\section{Isolated Calmness of the Solution Mapping}

In what follows we use the following notation. We denote by

$$
\bar{\Lambda}:=\left\{\lambda \in N_{D}(\tilde{g}(\bar{p}, \bar{x})) \mid b(\bar{p}, \bar{x})^{T} \lambda=-f(\bar{p}, \bar{x})\right\}
$$

the set of multipliers at our reference solution. we define for every $\lambda \in \mathbb{R}^{s}$ the Lagrangian $\mathscr{L}_{\lambda}(p, x): \mathbb{R}^{l} \times \mathbb{R}^{n} \rightarrow \mathbb{R}^{n}$ by

$$
\mathscr{L}_{\lambda}(p, x):=f(p, x)+b(p, x)^{T} \lambda .
$$

Definition 5.1 We say that the second-order condition for isolated calmness (SOCIC) holds at $(\bar{p}, \bar{x})$ if for every $u \neq 0$ and every $\lambda \in \tilde{\Lambda}((\bar{p}, \bar{x}),-f(\bar{p}, \bar{x}) ;(0, u))$ with

$$
\left.\nabla_{2} \tilde{g}(\bar{p}, \bar{x}) u \in \mathscr{K}_{D}(\tilde{g}(\bar{p}, \bar{x})), \lambda\right)
$$

there exists some $v \in \mathbb{R}^{n}$ such that

$$
b(\bar{p}, \bar{x}) v \in T_{\mathscr{K}_{D}(\tilde{g}(\bar{p}, \bar{x}), \lambda)}\left(\nabla_{2} \tilde{g}(\bar{p}, \bar{x}) u\right)
$$

and

$$
v^{T} \nabla_{2} \mathscr{L}_{\lambda}(\bar{p}, \bar{x}) u<0
$$

Theorem 5.2 Assume that Assumption 1 is fulfilled. If SOCIC holds at $(\bar{p}, \bar{x})$, then the solution map $S$ to the variational system (1.3) has the isolated calmness property at $(\bar{p}, \bar{x})$.

Conversely, if for every $u \neq 0$ there holds

$$
D G((\bar{p}, \bar{x}),-f(\bar{p}, \bar{x}))(0, u)=D \Psi((\bar{p}, \bar{x}, \bar{x}),-f(\bar{p}, \bar{x}))(0, u, u)
$$

and the mapping $M=f+G$ is metrically subregular in direction $(0, u)$ at $((\bar{p}, \bar{x}), 0)$, SOCIC is also necessary for the isolated calmness property of $S$ at $(\bar{p}, \bar{x})$. 
Proof We claim that SOCIC is equivalent to the condition

$$
0 \in \nabla f(p, x)(0, u)+D \Psi((\bar{p}, \bar{x}, \bar{x}),-f(\bar{p}, \bar{x}))(0, u, u) \Rightarrow u=0 .
$$

Assume on the contrary that there is some $u \neq 0$ such that

$$
0 \in \nabla f(p, x)(0, u)+D \Psi((\bar{p}, \bar{x}, \bar{x}),-f(\bar{p}, \bar{x}))(0, u, u) .
$$

By (4.26) this is equivalent to

$$
\begin{aligned}
0 & \in \nabla f(p, x)(0, u)+\nabla\left(b(\cdot)^{T} \lambda\right)(\bar{p}, \bar{x})(0, u)+b(\bar{p}, \bar{x})^{T} N_{\mathscr{K}_{D}(\tilde{g}(\bar{p}, \bar{x}), \lambda)}(\nabla \tilde{g}(\bar{p}, \bar{x})(0, u)) \\
& =\nabla_{2} \mathscr{L}_{\lambda}(\bar{p}, \bar{x}) u+b(\bar{p}, \bar{x})^{T} N_{\mathscr{K}_{D}(\tilde{g}(\bar{p}, \bar{x}), \lambda)}\left(\nabla_{2} \tilde{g}(\bar{p}, \bar{x}) u\right)
\end{aligned}
$$

for some $\lambda \in \tilde{\Lambda}((\bar{p}, \bar{x}),-f(\bar{x}) ;(0, u))$. In particular, $\nabla_{2} \tilde{g}(\bar{p}, \bar{x}) u \in \mathscr{K}_{D}(\tilde{g}(\bar{p}, \bar{x}), \lambda)$ follows. Next observe that

$$
\begin{aligned}
N_{\mathscr{K}_{D}(\tilde{g}(\bar{p}, \bar{x}), \lambda)}\left(\nabla_{2} \tilde{g}(\bar{p}, \bar{x}) u\right) & =\mathscr{K}_{D}(\tilde{g}(\bar{p}, \bar{x}), \lambda)^{\circ} \cap\left[\nabla_{2} \tilde{g}(\bar{p}, \bar{x}) u\right]^{\perp}=\left(\mathscr{K}_{D}(\tilde{g}(\bar{p}, \bar{x}), \lambda)+\left[\nabla_{2} \tilde{g}(\bar{p}, \bar{x}) u\right]\right)^{\circ} \\
& =\left(T_{\mathscr{K}_{D}(\tilde{g}(\bar{p}, \bar{x}), \lambda)}\left(\nabla_{2} \tilde{g}(\bar{p}, \bar{x}) u\right)\right)^{\circ}
\end{aligned}
$$

and thus

$$
b(\bar{p}, \bar{x})^{T} N_{\mathscr{K}_{D}(\tilde{g}(\bar{p}, \bar{x}), \lambda)}\left(\nabla_{2} \tilde{g}(\bar{p}, \bar{x}) u\right)=\left\{v \mid b(\bar{p}, \bar{x}) v \in T_{\mathscr{K}_{D}(\tilde{g}(\bar{p}, \bar{x}), \lambda)}\left(\nabla_{2} \tilde{g}(\bar{p}, \bar{x}) u\right)\right\}^{\circ} .
$$

This follows from [33, Corollary 16.3.2] because the set on the left hand side is a convex polyhedral set and therefore closed. Thus (5.33) is equivalent to

$$
-\nabla_{2} \mathscr{L}_{\lambda}(\bar{p}, \bar{x}) u \in\left\{v \mid b(\bar{p}, \bar{x}) v \in T_{\mathscr{K}_{D}(\tilde{g}(\bar{p}, \bar{x}), \lambda)}\left(\nabla_{2} \tilde{g}(\bar{p}, \bar{x}) u\right)\right\}^{\circ}
$$

which in turn is equivalent to

$$
-v^{T} \nabla_{2} \mathscr{L}_{\lambda}(\bar{p}, \bar{x}) u \leq 0 \forall v: b(\bar{p}, \bar{x}) v \in T_{\mathscr{K}_{D}(\tilde{g}(\bar{p}, \bar{x}), \lambda)}\left(\nabla_{2} \tilde{g}(\bar{p}, \bar{x}) u\right)
$$

contradicting (5.30). Thus the claimed equivalence between SOCIC and (5.32) holds true. Combining Theorem 3.1 and (4.25) we see that the condition (5.32) and consequently SOCIC as well are sufficient for the isolated calmness property of $S$ at $(\bar{p}, \bar{x})$.

In order to show the second statement of the theorem, just note that condition (5.31) ensures that (5.32) and SOCIC are equivalent to the condition

$$
0 \in \nabla f(p, x)(0, u)+D G((\bar{p}, \bar{x}, \bar{x}),-f(\bar{p}, \bar{x}))(0, u) \Rightarrow u=0
$$

and thus by Theorem 3.1 the necessity of SOCIC for the isolated calmness property of $S$ follows.

By Theorem 4.3(ii), a sufficient condition for (5.31) is that the system $\tilde{g}(\cdot) \in D$ is nondegenerate in every direction $(0, u), u \neq 0$ at $(\bar{p}, \bar{x})$. We now state a sufficient condition for the metric regularity of the mapping $M=f+G$ in some direction $(q, u)$.

Theorem 5.3 Let $(q, u) \in \mathbb{R}^{l} \times \mathbb{R}^{n}$ and assume that the system $\tilde{g}(\cdot) \in D$ is non-degenerate in direction $(q, u)$ at $(\bar{p}, \bar{x})$. Further assume that for every $\hat{\lambda} \in \Xi((\bar{p}, \bar{x}),-f(\bar{p}, \bar{x}) ;(q, u))$, every $\eta \in N_{\mathscr{K}_{D}(\tilde{g}(\bar{p}, \bar{x}), \hat{\lambda})}(\nabla \tilde{g}(\bar{p}, \bar{x})(q, u))$ satisfying $0=\nabla \mathscr{L}_{\hat{\lambda}}(\bar{p}, \bar{x})(q, u)+b(\bar{p}, \bar{x})^{T} \eta$, every pair of faces $\mathscr{F}_{1}, \mathscr{F}_{2}$ of the critical cone $\mathscr{K}_{D}(\tilde{g}(\bar{p}, \bar{x}), \hat{\lambda})$ with $\nabla \tilde{g}(\bar{p}, \bar{x})(q, u) \in$ $\mathscr{F}_{2} \subset \mathscr{F}_{1} \subset[\eta]^{\perp}$ and for every $0 \neq w \in \mathbb{R}^{n}$ with $b(\bar{p}, \bar{x}) w \in \mathscr{F}_{1}-\mathscr{F}_{2}$ there is some $(\tilde{q}, \tilde{u})$ such that $\nabla \tilde{g}(\bar{p}, \bar{x})(\tilde{q}, \tilde{u}) \in \mathscr{F}_{1}-\mathscr{F}_{2}$ and

$$
w^{T} \nabla \mathscr{L}_{\hat{\lambda}}(\bar{p}, \bar{x})(\tilde{q}, \tilde{u})>0 .
$$

Then the mapping $M$ is metrically regular in direction $((q, u), 0)$ at $((\bar{p}, \bar{x}), 0)$. 
Proof By contraposition. Assume on the contrary that $M=f+G$ is not metrically regular in direction $((q, u), 0)$ at $((\bar{p}, \bar{x}), 0)$. By virtue of Theorem 2.6 there is some $w \neq 0$ such that $(0,0) \in D^{*}(f+G)(((\bar{p}, \bar{x}), 0) ;((q, u), 0))(-w)$. In particular, this implies

$$
0 \in D M((\bar{p}, \bar{x}), 0)(q, u)=\nabla f(\bar{p}, \bar{x})(q, u)+D G((\bar{p}, \bar{x}),-f(\bar{p}, \bar{x}))(q, u) .
$$

By the definition of the directional limiting coderivative there are sequences $t_{k} \downarrow 0$, $\left(q_{k}, u_{k}, w_{k}^{*}\right) \rightarrow(q, u, 0)$ and $\left(q_{k}^{*}, u_{k}^{*}, w_{k}\right) \rightarrow(0,0, w)$ such that

$$
\left(q_{k}^{*}, u_{k}^{*}, w_{k}\right) \in \widehat{N}_{\mathrm{gph}(f+G)}\left(\left(p_{k}, x_{k}\right), t_{k} w_{k}^{*}\right),
$$

where $p_{k}:=\bar{p}+t_{k} q_{k}, x_{k}:=\bar{x}+t_{k} u_{k}$. Hence $\left(\left(q_{k}^{*}, u_{k}^{*}\right)+\nabla f\left(p_{k}, x_{k}\right)^{T} w_{k}, w_{k}\right) \in$ $\widehat{N}_{\mathrm{gph} G}\left(\left(p_{k}, x_{k}\right), x_{k}^{*}\right)$, where $x_{k}^{*}:=t_{k} w_{k}^{*}-f\left(p_{k}, x_{k}\right)$, which is equivalent to

$\left(q_{k}^{*}+\nabla_{1} f(\bar{p}, \bar{x})^{T} w_{k}\right)^{T} \sigma+\left(u_{k}^{*}+\nabla_{2} f(\bar{p}, \bar{x})^{T} w_{k}\right)^{T} \xi+w_{k}^{T} \xi^{*} \leq 0 \forall\left(\sigma, \xi, \xi^{*}\right) \in \operatorname{gph} D G\left(\left(p_{k}, x_{k}\right), x_{k}^{*}\right)$.

By Proposition 2.14, the system $\tilde{g}(\cdot) \in D$ is non-degenerate at $\left(p_{k}, x_{k}\right)$ and we deduce from Theorem 4.3 that $D G\left(\left(p_{k}, x_{k}\right), x_{k}^{*}\right)\left(q^{\prime}, u^{\prime}\right)=D \Psi\left(\left(p_{k}, x_{k}\right), x_{k}^{*}\right)\left(q^{\prime}, u^{\prime}, u^{\prime}\right) \forall\left(q^{\prime}, u^{\prime}\right) \in$ $\mathbb{R}^{l} \times \mathbb{R}^{n}$. Hence, by taking $\sigma=0, \xi=0$ we obtain

$$
w_{k}^{T} b\left(p_{k}, x_{k}\right)^{T} \zeta^{*} \leq 0 \forall \zeta^{*} \in \mathscr{K}_{D}\left(\tilde{g}\left(p_{k}, x_{k}\right), \lambda\right)^{\circ}, \lambda \in \tilde{\Lambda}\left(\left(p_{k}, x_{k}\right), x_{k}^{*},(0,0)\right) .
$$

Since $\tilde{\Lambda}\left(\left(p_{k}, x_{k}\right), x_{k}^{*} ;(0,0)\right)=\left\{\lambda \in N_{D}\left(\tilde{g}\left(p_{k}, x_{k}\right)\right) \mid b\left(p_{k}, x_{k}\right)^{T} \lambda=x_{k}^{*}\right\}$ and $x_{k}^{*} \in$ $G\left(p_{k}, x_{k}\right)$, by Assumption 1 there exists for every $k$ some $\lambda_{k} \in \tilde{\Lambda}\left(\left(p_{k}, x_{k}\right), x_{k}^{*},(0,0)\right) \cap$ $\kappa\left\|x_{k}^{*}\right\| \mathscr{B}_{\mathbb{R}^{s}}$. By passing to a subsequence if necessary we can assume that $\lambda_{k}$ converges to some $\hat{\lambda}$. Obviously we have $\hat{\lambda} \in N_{D}(\tilde{g}(\bar{p}, \bar{x}))$ and $b(\bar{p}, \bar{x})^{T} \hat{\lambda}=-f(\bar{p}, \bar{x})$. By [5, Lemma 4H.2], for each $k$ sufficiently large there are two closed faces $\mathscr{F}_{2}^{k} \subset \mathscr{F}_{1}^{k}$ of the critical cone $\mathscr{K}_{D}(\tilde{g}(\bar{p}, \bar{x}), \hat{\lambda})$ such that $\mathscr{K}_{D}\left(\tilde{g}\left(p_{k}, x_{k}\right), \lambda_{k}\right)=\mathscr{F}_{1}^{k}-\mathscr{F}_{2}^{k}$ and a close look at the proof of [5, Lemma 4H.2] tells us that we also have $\tilde{g}\left(p_{k}, x_{k}\right)-\tilde{g}(\bar{p}, \bar{x}) \in$ ri $\mathscr{F}_{2}^{k}$. Since $\mathscr{K}_{D}(\tilde{g}(\bar{p}, \bar{x}), \hat{\lambda})$ is a closed convex cone, it has only finitely many faces and by passing to a subsequence once more we can assume $\mathscr{F}_{1}^{k}=\mathscr{F}_{1}$ and $\mathscr{F}_{2}^{k}=\mathscr{F}_{2}$ for all $k$. A face of a closed convex cone is again a cone and thus $\left(\tilde{g}\left(p_{k}, x_{k}\right)-\tilde{g}(\bar{p}, \bar{x})\right) / t_{k} \in$ ri $\mathscr{F}_{2} \forall k$. This yields by passing to the limit that $\nabla \tilde{g}(\bar{p}, \bar{x})(q, u) \in \mathscr{F}_{2} \subset \mathscr{K}_{D}(\bar{g}(\bar{p}, \bar{x}), \hat{\lambda})$, and consequently $\hat{\lambda} \in \Xi((\bar{p}, \bar{x}),-f(\bar{p}, \bar{x}),(q, u))$.

$$
\begin{aligned}
t_{k} w_{k}^{*}-f\left(p_{k}, x_{k}\right) & =x_{k}^{*}=b\left(p_{k}, x_{k}\right)^{T} \lambda_{k}=b(\bar{p}, \bar{x})^{T} \lambda_{k}+t_{k} \nabla\left(b(\cdot)^{T} \lambda_{k}\right)(\bar{p}, \bar{x})\left(q_{k}, u_{k}\right)+o\left(t_{k}\right) \\
& =-f(\bar{p}, \bar{x})+b(\bar{p}, \bar{x})^{T}\left(\lambda_{k}-\hat{\lambda}\right)+t_{k} \nabla\left(b(\cdot)^{T} \hat{\lambda}\right)(\bar{p}, \bar{x})(q, u)+o\left(t_{k}\right),
\end{aligned}
$$

yielding

$$
\begin{aligned}
b(\bar{p}, \bar{x})^{T} \frac{\lambda_{k}-\hat{\lambda}}{t_{k}} & =w_{k}^{*}-\frac{f\left(p_{k}, x_{k}\right)-f(\bar{p}, \bar{x})}{t_{k}}-\nabla\left(b(\cdot)^{T} \hat{\lambda}\right)(\bar{p}, \bar{x})(q, u)+o\left(t_{k}\right) / t_{k} \\
& =w_{k}^{*}-\nabla \mathscr{L}_{\hat{\lambda}}(q, u)+o\left(t_{k}\right) / t_{k} .
\end{aligned}
$$

Since $\lambda_{k} \in N_{D}\left(\tilde{g}\left(p_{k}, x_{k}\right)\right) \subset N_{D}(\tilde{g}(\bar{p}, \bar{x}))$, it holds that $\lambda_{k}-\hat{\lambda}$ and consequently $\frac{\lambda_{k}-\hat{\lambda}}{t_{k}}$ belong to $T_{N_{D}(\tilde{g}(\bar{p}, \bar{x}))}(\hat{\lambda})$. Because of $\nabla \tilde{g}(\bar{p}, \bar{x})(q, u) \in \mathscr{F}_{2} \subset \mathscr{F}_{1}$ we conclude 
$\nabla \tilde{g}(\bar{p}, \bar{x})(q, u) \in \mathscr{F}_{1}-\mathscr{F}_{2}=\mathscr{K}_{D}\left(\tilde{g}\left(p_{k}, x_{k}\right), \lambda_{k}\right)$ showing $\lambda_{k}^{T} \nabla \tilde{g}(\bar{p}, \bar{x})(q, u)=0$. Together with $\hat{\lambda}^{T} \nabla \tilde{g}(\bar{p}, \bar{x})(q, u)=0$ we obtain

$$
\begin{aligned}
\frac{\lambda_{k}-\hat{\lambda}}{t_{k}} & \in T_{N_{D}(\tilde{g}(\bar{p}, \bar{x}))}(\hat{\lambda}) \cap[\nabla \tilde{g}(\bar{p}, \bar{x})(q, u)]^{\perp}=\left(N_{D}(\tilde{g}(\bar{p}, \bar{x}))+[\hat{\lambda}]\right) \cap[\nabla \tilde{g}(\bar{p}, \bar{x})(q, u)]^{\perp} \\
& =\left(T_{D}(\tilde{g}(\bar{p}, \bar{x})) \cap[\hat{\lambda}]^{\perp}\right)^{\circ} \cap[\nabla \tilde{g}(\bar{p}, \bar{x})(q, u)]^{\perp}=N_{\mathscr{K}_{D}(\tilde{g}(\bar{p}, \bar{x}), \hat{\lambda})}(\nabla \tilde{g}(\bar{p}, \bar{x})(q, u)) .
\end{aligned}
$$

Since $\mathscr{F}_{1}-\mathscr{F}_{2}=\mathscr{K}_{D}\left(\tilde{g}\left(p_{k}, x_{k}\right), \lambda_{k}\right) \subset\left[\lambda_{k}\right]^{\perp}$ and $\mathscr{F}_{2} \subset \mathscr{F}_{1} \subset \mathscr{K}_{D}(\tilde{g}(\bar{p}, \bar{x}), \hat{\lambda})$, we have

$$
\mathscr{F}_{1}-\mathscr{F}_{2} \subset\left[\lambda_{k}\right]^{\perp} \cap[\hat{\lambda}]^{\perp}=\left(\left[\lambda_{k}\right]+[\hat{\lambda}]\right)^{\perp} \subset\left[\lambda_{k}-\hat{\lambda}\right]^{\perp}
$$

and consequently $\left[\lambda_{k}-\hat{\lambda}\right] \subset\left(\operatorname{sp} \mathscr{F}_{1}\right)^{\perp}$. We can now invoke Hoffman's lemma [3, Theorem $2.200]$ to find for every $k$ some $\eta_{k} \in N_{\mathscr{K}_{D}(\tilde{g}(\bar{p}, \bar{x}), \hat{\lambda})}(\nabla \tilde{g}(\bar{p}, \bar{x})(q, u)) \cap\left(\operatorname{sp} \mathscr{F}_{1}\right)^{\perp}$ satisfying

$$
b(\bar{p}, \bar{x})^{T} \frac{\lambda_{k}-\hat{\lambda}}{t_{k}}=b(\bar{p}, \bar{x})^{T} \eta_{k}
$$

and $\left\|\eta_{k}\right\| \leq \beta\left\|b(\bar{p}, \bar{x})^{T}\left(\lambda_{k}-\hat{\lambda}\right) / t_{k}\right\|$ for some constant $\beta>0$ not depending on $k$. Since the right hand side of (5.36) is bounded, so is $\eta_{k}$ and by possibly passing to a subsequence we can assume that $\eta_{k}$ converges to some $\eta \in N_{\mathscr{K}_{D}(\tilde{g}(\bar{p}, \bar{x}), \hat{\lambda})}(\nabla \tilde{g}(\bar{p}, \bar{x})(q, u)) \cap\left(\operatorname{sp} \mathscr{F}_{1}\right)^{\perp}$ satisfying

$$
b(\bar{p}, \bar{x})^{T} \eta=\lim _{k \rightarrow \infty}\left(-\nabla \mathscr{L}_{\hat{\lambda}}(\bar{p}, \bar{x})(q, u)+o\left(t_{k}\right) / t_{k}+w_{k}^{*}\right)=-\nabla \mathscr{L}_{\hat{\lambda}}(\bar{p}, \bar{x})(q, u) .
$$

From $\eta \in\left(\operatorname{sp} \mathscr{F}_{1}\right)^{\perp}$ we conclude $\mathscr{F}_{1} \subset \operatorname{sp} \mathscr{F}_{1} \subset[\eta]^{\perp}$. Moreover, by passing $k$ to infinity in (5.35) it follows that

$$
w^{T} b(\bar{p}, \bar{x})^{T} \zeta^{*} \leq 0 \forall \zeta^{*} \in\left(\mathscr{F}_{1}-\mathscr{F}_{2}\right)^{\circ},
$$

which is the same as $b(\bar{p}, \bar{x}) w \in \mathscr{F}_{1}-\mathscr{F}_{2}$. By the assumption of the theorem there is some $(\tilde{q}, \tilde{u})$ with $\nabla \tilde{g}(\bar{p}, \bar{x})(\tilde{q}, \tilde{u}) \in \mathscr{F}_{1}-\mathscr{F}_{2}$ and $w^{T} \nabla \mathscr{L}_{\hat{\lambda}}(\bar{p}, \bar{x})(\tilde{q}, \tilde{u})>0$. Applying Corollary 2.12 we obtain

$$
\operatorname{sp} N_{T_{D}(\tilde{g}(\bar{p}, \bar{x}))}(\nabla \tilde{g}(\bar{p}, \bar{x})(q, u)) \supset\left(\mathscr{F}_{2}-\mathscr{F}_{2}\right)^{\circ} \supset\left(\mathscr{F}_{1}-\mathscr{F}_{2}\right)^{\circ}
$$

implying the condition

$$
\nabla \tilde{g}(\bar{p}, \bar{x})^{T} \mu=0, \mu \in\left(\mathscr{F}_{1}-\mathscr{F}_{2}\right)^{\circ} \Rightarrow \mu=0 .
$$

From Theorem 2.8 we can deduce that for every $k$ there is some $\left(\tilde{q}_{k}, \tilde{u}_{k}\right)$ satisfying

$$
\nabla \tilde{g}\left(p_{k}, x_{k}\right)\left(\tilde{q}_{k}, \tilde{u}_{k}\right) \in \mathscr{F}_{1}-\mathscr{F}_{2}=\mathscr{K}_{D}\left(\tilde{g}\left(p_{k}, x_{k}\right), \lambda_{k}\right)
$$

and

$\left\|\left(\tilde{q}_{k}, \tilde{u}_{k}\right)-(\tilde{q}, \tilde{u})\right\| \leq \beta^{\prime} \operatorname{dist}\left(\nabla \tilde{g}\left(p_{k}, x_{k}\right)(\tilde{q}, \tilde{u}), \mathscr{F}_{1}-\mathscr{F}_{2}\right) \leq \beta^{\prime}\left\|\left(\nabla \tilde{g}\left(p_{k}, x_{k}\right)-\nabla \tilde{g}(\bar{p}, \bar{x})\right)(\tilde{q}, \tilde{u})\right\|$ for some constant $\beta^{\prime} \geq 0$ not depending on $k$. Since $\tilde{g}(\cdot) \in D$ is non-degenerate at $\left(p_{k}, x_{k}\right)$ by Proposition 2.14, we obtain $\Lambda\left(\left(p_{k}, x_{k}, x_{k}\right), \nabla g\left(p_{k}, x_{k}, x_{k}\right)^{T} \lambda_{k} ;\left(\tilde{q}_{k}, \tilde{u}_{k}, \tilde{u}_{k}\right)\right)=\left\{\lambda_{k}\right\}$ by Theorem 4.3 and thus

$$
\left(\left(\tilde{q}_{k}, \tilde{u}_{k}\right), \nabla\left(b(\cdot)^{T} \lambda_{k}\right)\left(p_{k}, x_{k}\right)\left(\tilde{q}_{k}, \tilde{u}_{k}\right)\right) \in \operatorname{gph} D G\left(\left(p_{x}, x_{k}\right), x_{k}^{*}\right) .
$$

Hence we obtain from (5.34)

$$
\begin{gathered}
\left(q_{k}^{*}+\nabla_{1} f\left(p_{k}, x_{k}\right)^{T} w_{k}\right)^{T} \tilde{q}_{k}+\left(u_{k}^{*}+\nabla_{2} f\left(p_{k}, x_{k}\right)^{T} w_{k}\right)^{T} \tilde{u}_{k}+w_{k}^{T} \nabla\left(b(\cdot)^{T} \lambda_{k}\right)\left(p_{k}, x_{k}\right)\left(\tilde{q}_{k}, \tilde{u}_{k}\right) \\
=q_{k}^{* T} \tilde{q}_{k}+u_{k}^{* T} \tilde{u}_{k}+w_{k}^{T} \nabla \mathscr{L}_{\lambda_{k}}\left(p_{k}, x_{k}\right)\left(\tilde{q}_{k}, \tilde{u}_{k}\right) \leq 0 .
\end{gathered}
$$


By passing $k$ to infinity this yields the contradiction $w^{T} \nabla \mathscr{L}_{\hat{\lambda}}(\bar{p}, \bar{x})(\tilde{q}, \tilde{u}) \leq 0$ and hence $M$ is metrically regular in direction $((q, u), 0)$ at $((\bar{p}, \bar{x}), 0)$.

In case when $(q, u)=(0,0)$ Theorem 5.3 constitutes a sufficient condition for the metric regularity of $M$ around $((\bar{p}, \bar{x}), 0)$. This is an interesting result for its own sake. On the other hand, when applying Theorem 5.3 for directions $(0, u), u \neq 0$, we have an efficient tool for verifying the necessity of SOCIC for the isolated calmness property of $S$.

Remark 5.4 Condition (5.31) and the requirement that $M$ is metrically subregular are fulfilled in particular in case of canonical perturbations, i.e., parametric systems given by (1.3) with $p=\left(p_{1}, p_{2}\right) \in \mathbb{R}^{n} \times \mathbb{R}^{s}, f(p, x)=\hat{f}(x)-p_{1}$ and $\tilde{g}(p, x)=\hat{g}(x)-p_{2}$.

Example 5.5 Consider the variational system (1.3) with $D:=\mathbb{R}_{-}^{2}$ and $f: \mathbb{R}^{2} \times \mathbb{R}^{2} \rightarrow \mathbb{R}^{2}$, $g: \mathbb{R}^{2} \times \mathbb{R}^{2} \times \mathbb{R}^{2} \rightarrow \mathbb{R}^{2}$ given by

$$
f(p, x):=\left(\begin{array}{c}
x_{1}-p_{1} \\
-x_{2}
\end{array}\right), \quad g(p, x, z):=\left(\begin{array}{c}
p_{2}-x_{1}+x_{2}+z_{2} \\
-x_{1}-3 x_{2}+z_{2}
\end{array}\right)
$$

at $\bar{p}=\bar{x}=(0,0)$. Condition (4.24) ensuring Assumption 1 reads as

$$
\left(\begin{array}{c}
0 \\
\mu_{1}+\mu_{2}
\end{array}\right)=\left(\begin{array}{l}
0 \\
0
\end{array}\right), \mu_{1}, \mu_{2} \geq 0 \Rightarrow \mu_{1}=\mu_{2}=0
$$

and is certainly fulfilled. Further,

$$
b(p, x)=\left(\begin{array}{ll}
0 & 1 \\
0 & 1
\end{array}\right), \quad \tilde{g}(p, x)=\left(\begin{array}{c}
p_{2}-x_{1}+2 x_{2} \\
-x_{1}-2 x_{2}
\end{array}\right)
$$

and for each $p \in \mathbb{R}^{2}$ the solution set $S(p)$ consists of those $x$ such that there exists some $\lambda \in N_{\mathbb{R}_{-}^{2}}(\tilde{g}(p, x))$ fulfilling

$$
0=\mathscr{L}_{\lambda}(p, x)=\left(\begin{array}{c}
x_{1}-p_{1} \\
-x_{2}+\lambda_{1}+\lambda_{2}
\end{array}\right) .
$$

Straightforward calculations yield that the solution map $S$ is given by

$$
S(p)= \begin{cases}\left\{\left(p_{1}, 0\right),\left(p_{1}, \frac{p_{1}-p_{2}}{2}\right)\right\} & \text { if } p_{2}-p_{1} \leq 0, p_{1} \geq 0, \\ \left\{\left(p_{1},-\frac{p_{1}}{2}\right),\left(p_{1}, \frac{p_{1}-p_{2}}{2}\right)\right\} & \text { if } p_{2}-2 p_{1} \leq 0, p_{1}<0, \\ \emptyset & \text { otherwise. }\end{cases}
$$

We see that $S$ has the isolated calmness property at $(\bar{p}, \bar{x})$ and we now want to verify that SOCIC is fulfilled. Consider $u \neq 0$ such that

$$
\nabla_{2} \tilde{g}(\bar{p}, \bar{x}) u=\left(\begin{array}{l}
-u_{1}+2 u_{2} \\
-u_{1}-2 u_{2}
\end{array}\right) \in T_{D}(\tilde{g}(\bar{p}, \bar{x}))=\mathbb{R}_{-}^{2} .
$$

In particular we have $u_{1} \neq 0$ because $u_{1}=0$ implies $u_{2}=0$ and the case $u=0$ is excluded. Since $\Xi((\bar{p}, \bar{x}),-f(\bar{p}, \bar{x}))=\left\{\mu \in \mathbb{R}_{+}^{2} \mid\left(0, \mu_{1}+\mu_{2}\right)=(0,0)\right\}=\{(0,0)\}$, we have $\Xi((\bar{p}, \bar{x}),-f(\bar{p}, \bar{x}) ;(0, u))=\tilde{\Lambda}((\bar{p}, \bar{x}),-f(\bar{p}, \bar{x}) ;(0, u))=\{(0,0)\}$. By choosing $v=\left(-u_{1}, 0\right)$ we have $b(\bar{p}, \bar{x}) v=0 \in T_{\mathscr{K}_{D}(\tilde{g}(\bar{p}, \bar{x}), \lambda)}\left(\nabla_{2} \tilde{g}(\bar{p}, \bar{x}) u\right)$ and $v^{T} \nabla_{2} \mathscr{L}_{0}(\bar{p}, \bar{x}) u=$ $-u_{1}^{2}<0$ and SOCIC is established.

Next we show that the mapping $(p, x) \rightrightarrows M(p, x)=f(p, x)+G(p, x)$ is metrically regular around $((\bar{p}, \bar{x}), 0)$ by applying Theorem 5.3 with $(q, u)=(0,0)$. The Jacobian $\nabla \tilde{g}(\bar{p}, \bar{x})$ has full row rank and hence the system $\tilde{g}(\cdot) \in \mathbb{R}_{-}^{2}$ is non-degenerate. It can be easily deduced that the only $\hat{\lambda} \in \Xi((\bar{p}, \bar{x}),-f(\bar{p}, \bar{x}) ;(0,0))$ and the only 
$\eta \in N_{\mathscr{K}_{D}(\tilde{g}(\bar{p}, \bar{x}), \hat{\lambda})}(\nabla \tilde{g}(\bar{p}, \bar{x})(0,0))$ satisfying $0=\nabla \mathscr{L}_{\hat{\lambda}}(\bar{p}, \bar{x})(0,0)+b(\bar{p}, \bar{x})^{T} \eta$ are $\hat{\lambda}=\eta=(0,0)$. We have to show that for every pair of faces $\mathscr{F}_{2} \subset \mathscr{F}_{1} \subset \mathbb{R}_{-}^{2}$ and every $0 \neq w \in \mathbb{R}^{2}$ satisfying $b(\bar{p}, \bar{x}) w=\left(w_{2}, w_{2}\right)^{T} \in \mathscr{F}_{1}-\mathscr{F}_{2}$ there is some $(\tilde{q}, \tilde{u})$ with

$\nabla \tilde{g}(\bar{p}, \bar{x})(\tilde{q}, \tilde{u})=\left(\begin{array}{c}\tilde{q}_{2}-\tilde{u}_{1}+2 \tilde{u}_{2} \\ -\tilde{u}_{1}-2 \tilde{u}_{2}\end{array}\right) \in \mathscr{F}_{1}-\mathscr{F}_{2}$ and $w^{T} \nabla \mathscr{L}_{0}(\bar{p}, \bar{x})(\tilde{q}, \tilde{u})=w_{1}\left(\tilde{u}_{1}-\tilde{q}_{1}\right)-w_{2} \tilde{u}_{2}>0$.

If $w_{1} \neq 0$, this can be easily accomplished by taking $\tilde{q}_{2}=\tilde{u}_{1}=\tilde{u}_{2}=0$ and $\tilde{q}_{1}=-w_{1}$. So let $w_{1}=0$ and consequently $w_{2} \neq 0$. Then condition (5.38) is fulfilled when we take, e.g., $\tilde{u}_{2}=-w_{2}, \tilde{u}_{1}=-2 \tilde{u}_{2}, \tilde{q}_{2}=\tilde{u}_{1}-2 \tilde{u}_{2}$ and an arbitrary $\tilde{q}_{1}$. So, we have detected the metric regularity of $M$ from Theorem 5.3.

\section{On the Aubin Property of the Solution Map}

In the following theorem we state our main result concerning the Aubin property of the solution map $S$ relative to some set $P$. It improves [18, Theorem 6], because Assumption 1 is weaker than Assumption (A) in [18] and we use here a less restrictive non-degeneracy condition.

Theorem 6.1 Assume that Assumption 1 is fulfilled and we are given a closed set $P \subset \mathbb{R}^{l}$ containing $\bar{p}$ such that the following assumptions are fulfilled:

(i) For every $q \in T_{P}(\bar{p})$ there is some $u \in \mathbb{R}^{n}$ such that

$$
0 \in \nabla f(\bar{p}, \bar{x})(q, u)+D \Psi((\bar{p}, \bar{x}, \bar{x}),-f(\bar{p}, \bar{x}))(q, u, u) .
$$

(ii) For every $(0,0) \neq(q, u)$ verifying (6.39) the (partial) directional non-degeneracy condition

$$
\nabla_{2} \tilde{g}(\bar{p}, \bar{x})^{T} \mu=0, \mu \in \operatorname{sp} N_{T_{D}(\tilde{g}(\bar{p}, \bar{x}))}(\nabla \tilde{g}(\bar{p}, \bar{x})(q, u)) \Rightarrow \mu=0
$$

is fulfilled and for every $\hat{\lambda} \in \Xi((\bar{p}, \bar{x}),-f(\bar{p}, \bar{x}) ;(q, u))$, every $\eta \in$ $N_{\mathscr{K}_{D}(\tilde{g}(\bar{p}, \bar{x}), \hat{\lambda})}(\nabla \tilde{g}(\bar{p}, \bar{x})(q, u))$ satisfying $0=\nabla \mathscr{L}_{\hat{\lambda}}(\bar{p}, \bar{x})(q, u)+b(\bar{p}, \bar{x})^{T} \eta$, every pair of faces $\mathscr{F}_{1}, \mathscr{F}_{2}$ of the critical cone $\mathscr{K}_{D}(\tilde{g}(\bar{p}, \bar{x}), \hat{\lambda})$ with $\nabla \tilde{g}(\bar{p}, \bar{x})(q, u) \in$ $\mathscr{F}_{2} \subset \mathscr{F}_{1} \subset[\eta]^{\perp}$ and every $w \neq 0$ with $b(\bar{p}, \bar{x}) w \in \mathscr{F}_{1}-\mathscr{F}_{2}$ there is some $\tilde{w}$ with $\nabla_{2} \tilde{g}(\bar{p}, \bar{x}) \tilde{w} \in \mathscr{F}_{1}-\mathscr{F}_{2}$ such that

$$
w^{T} \nabla_{2} \mathscr{L}_{\hat{\lambda}}(\bar{p}, \bar{x}) \tilde{w}>0 .
$$

Then the solution mapping $S$ to the variational system (1.3) has the Aubin property relative to $P$ around $(\bar{p}, \bar{x})$ and for every $q \in T_{P}(\bar{p})$ there holds

$$
D S(\bar{p}, \bar{x})(q)=\{u \mid 0 \in \nabla f(\bar{p}, \bar{x})(q, u)+D \Psi((\bar{p}, \bar{x}, \bar{x}),-f(\bar{p}, \bar{x}))(q, u, u)\} .
$$

Proof We will invoke Theorem 3.5 in order to prove the proposition. Observe that (6.40) implies the non-degeneracy of the system $\tilde{g}(\cdot) \in D$ in direction $(q, u)$ at $(\bar{p}, \bar{x})$ and by Theorem 4.3 we have that $D \Psi((\bar{p}, \bar{x}, \bar{x}),-f(\bar{p}, \bar{x}))(q, u, u)=$ $D G((\bar{p}, \bar{x}),-f(\bar{p}, \bar{x}))(q, u)$ and all tangents $\left(q, u, u^{*}\right)$ to gph $G$ at $((\bar{p}, \bar{x}),-f(\bar{p}, \bar{x}))$ are derivable. Since $D M((\bar{p}, \bar{x}), 0)(q, u)=\nabla f(\bar{p}, \bar{x})(q, u)+D G((\bar{p}, \bar{x}),-f(\bar{p}, \bar{x}))(q, u)$ and taking into account Remark 3.7, assumption (i) of Theorem 3.5 is satisfied due to the first assumption. 
We now show that assumption (ii) of Theorem 3.5 is fulfilled as well. Assume that we are given a direction $(0,0) \neq(q, u)$ satisfying

$$
\begin{aligned}
0 & \in D M((\bar{p}, \bar{x}), 0)(q, u)=\nabla f(\bar{p}, \bar{x})(q, u)+D G((\bar{p}, \bar{x}),-f(\bar{p}, \bar{x}))(q, u) \\
& \subset \nabla f(\bar{p}, \bar{x})(q, u)+D \Psi((\bar{p}, \bar{x}, \bar{x}),-f(\bar{p}, \bar{x}))(q, u, u)
\end{aligned}
$$

and $\left(q^{*}, w\right)$ such that $\left(q^{*}, 0\right) \in D^{*} M(((\bar{p}, \bar{x}), 0) ;((q, u), 0))(-w)$.

By the definition of the directional limiting coderivative there are sequences $t_{k} \downarrow 0$, $\left(q_{k}, u_{k}, w_{k}^{*}\right) \rightarrow(q, u, 0)$ and $\left(q_{k}^{*}, u_{k}^{*}, w_{k}\right) \rightarrow\left(q^{*}, 0, w\right)$ such that

$$
\left(q_{k}^{*}, u_{k}^{*}, w_{k}\right) \in \widehat{N}_{\mathrm{gph}(f+G)}\left(\left(p_{k}, x_{k}\right), t_{k} w_{k}^{*}\right),
$$

where $p_{k}:=\bar{p}+t_{k} q_{k}, x_{k}:=\bar{x}+t_{k} u_{k}$. We can now proceed as in the proof of Theorem 5.3 to find the sequences $x_{k}^{*}$ and $\lambda_{k}$ as well as

$$
\hat{\lambda}=\lim _{k \rightarrow \infty} \lambda_{k} \in \Xi((\bar{p}, \bar{x}),-f(\bar{p}, \bar{x}) ;(q, u)), \eta \in N_{\mathscr{K}_{D}(\tilde{g}(\bar{p}, \bar{x}), \hat{\lambda})}(\nabla \tilde{g}(\bar{p}, \bar{x})(q, u))
$$

with $0=\nabla \mathscr{L}_{\hat{\lambda}}(\bar{p}, \bar{x})(q, u)+b(\bar{p}, \bar{x})^{T} \eta$ and the faces $\mathscr{F}_{1}, \mathscr{F}_{2}$ of the critical cone $\mathscr{K}_{D}(\tilde{g}(\bar{p}, \bar{x}), \hat{\lambda})$ with $\nabla \tilde{g}(\bar{p}, \bar{x})(q, u) \subset \mathscr{F}_{2} \subset \mathscr{F}_{1} \subset[\eta]^{\perp}$ such that $\left.\mathscr{K}_{D}\left(\tilde{g}\left(p_{k}, x_{k}\right), \lambda_{k}\right)\right)=$ $\mathscr{F}_{1}-\mathscr{F}_{2} \forall k$. As in the proof of Theorem 5.3 we can also deduce $b(\bar{p}, \bar{x}) w \in \mathscr{F}_{1}-\mathscr{F}_{2}$. By assumption (ii) of the theorem there is some $\tilde{w}$ with $\nabla_{2} \tilde{g}(\bar{p}, \bar{x}) \tilde{w} \in \mathscr{F}_{1}-\mathscr{F}_{2}$ and $w^{T} \nabla_{2} \mathscr{L}_{\hat{\lambda}}(\bar{p}, \bar{x}) \tilde{w}>0$, provided $w \neq 0$, which we now assume.

Next observe that the implication

$$
\nabla_{2} g(\bar{p}, \bar{x})^{T} \mu=0, \mu \in\left(\mathscr{F}_{1}-\mathscr{F}_{2}\right)^{\circ} \Rightarrow \mu=0
$$

follows from (6.40) by virtue of Corollary 2.12. By condition (6.41) and Theorem 2.8, there is some real $\beta>0$ such that for every $k$ sufficiently large there are some $\tilde{w}_{k}$ satisfying $\nabla_{2} \tilde{g}\left(p_{k}, x_{k}\right) \tilde{w}_{k} \in \mathscr{F}_{1}-\mathscr{F}_{2}$ and

$$
\left\|\tilde{w}_{k}-\tilde{w}\right\| \leq \beta \operatorname{dist}\left(\nabla_{2} \tilde{g}\left(p_{k}, x_{k}\right) \tilde{w}, \mathscr{F}_{1}-\mathscr{F}_{2}\right) \leq \beta\left\|\left(\nabla_{2} \tilde{g}\left(p_{k}, x_{k}\right)-\nabla_{2} \tilde{g}(\bar{p}, \bar{x})\right) \tilde{w}\right\| .
$$

Hence $\nabla \tilde{g}\left(p_{k}, x_{k}\right)\left(0, \tilde{w}_{k}\right) \in \mathscr{F}_{1}-\mathscr{F}_{2}=\mathscr{K}_{D}\left(\tilde{g}\left(p_{k}, x_{k}\right), \lambda_{k}\right)$ and, since $\tilde{g}(\cdot) \in D$ is nondegenerate at $\left(p_{k}, x_{k}\right)$, we obtain $\Lambda\left(\left(p_{k}, x_{k}, x_{k}\right), x_{k}^{*} ;\left(0, \tilde{w}_{k}, \tilde{w}_{k}\right)\right)=\left\{\lambda_{k}\right\}$ by Theorem 4.3. Using Theorem 4.3 once more together with (4.26) we obtain

$$
\left(0, \tilde{w}_{k}, \nabla\left(b(\cdot)^{T} \lambda_{k}\right)\left(p_{k}, x_{k}\right)\left(0, \tilde{w}_{k}\right)\right) \in \operatorname{gph} D G\left(\left(p_{k}, x_{k}\right), x_{k}^{*}\right),
$$

yielding

$\left(u_{k}^{*}+\nabla_{2} f\left(p_{k}, x_{k}\right)^{T} w_{k}\right)^{T} \tilde{w}_{k}+w_{k}^{T} \nabla_{2}\left(b(\cdot)^{T} \lambda_{k}\right)\left(p_{k}, x_{k}\right) \tilde{w}_{k}=u_{k}^{* T} \tilde{w}_{k}+w_{k}^{T} \nabla_{2} \mathscr{L}_{\lambda_{k}}\left(p_{k}, x_{k}\right) \tilde{w}_{k} \leq 0$

from (5.34). By passing to the limit we obtain the contradiction $w^{T} \nabla_{2} \mathscr{L}_{\hat{\lambda}} \tilde{w} \leq 0$ and thus $w=0$. It remains to show that $q^{*}=0$. Observe that (6.41) is equivalent to

$$
\left(\operatorname{ker} \nabla_{2} g(\bar{p}, \bar{x})^{T} \cap\left(\mathscr{F}_{1}-\mathscr{F}_{2}\right)^{\circ}\right)^{\circ}=\nabla_{2} g(\bar{p}, \bar{x}) \mathbb{R}^{n}+\left(\mathscr{F}_{1}-\mathscr{F}_{2}\right)=\{0\}^{\circ}=\mathbb{R}^{s} .
$$

Hence there is some $\bar{u} \in \mathbb{R}^{n}$ with $\nabla_{1} \tilde{g}(\bar{p}, \bar{x}) q^{*}+\nabla_{2} \tilde{g}(\bar{p}, \bar{x}) \bar{u} \in \mathscr{F}_{1}-\mathscr{F}_{2}$. Further, by assumption (6.41) and Theorem 2.8, there is some real $\beta>0$ such that for every $k$ sufficiently large there exist some vectors $\bar{u}_{k}$ satisfying $\nabla_{1} \tilde{g}\left(p_{k}, x_{k}\right) q^{*}+\nabla_{2} \tilde{g}\left(p_{k}, x_{k}\right) \bar{u}_{k} \in \mathscr{F}_{1}-$ $\mathscr{F}_{2}$ and $\left\|\bar{u}_{k}-\bar{u}\right\| \leq \beta \operatorname{dist}\left(\nabla_{1} \tilde{g}\left(p_{k}, x_{k}\right) q^{*}+\nabla_{2} \tilde{g}\left(p_{k}, x_{k}\right) \bar{u}, \mathscr{F}_{1}-\mathscr{F}_{2}\right) \leq \beta \|\left(\nabla \tilde{g}\left(p_{k}, x_{k}\right)-\right.$ $\nabla \tilde{g}(\bar{p}, \bar{x}))\left(q^{*}, \bar{u}\right) \|$. Using similar arguments as before we deduce

$$
\left(q^{*}, \bar{u}_{k}, \nabla\left(b(\cdot)^{T} \lambda_{k}\right)\left(p_{k}, x_{k}\right)\left(q^{*}, \bar{u}_{k}\right)\right) \in \operatorname{gph} D G\left(\left(p_{k}, x_{k}\right), x_{k}^{*}\right),
$$


resulting in

$$
\begin{aligned}
& \left(q_{k}^{*}+\nabla_{1} f\left(p_{k}, x_{k}\right)^{T} w_{k}\right)^{T} q^{*}+\left(u_{k}^{*}+\nabla_{2} f\left(p_{k}, x_{k}\right)^{T} w_{k}\right)^{T} \bar{u}_{k}+w_{k}^{T} \nabla\left(b(\cdot)^{T} \lambda_{k}\right)\left(p_{k}, x_{k}\right)\left(q^{*}, \bar{u}_{k}\right) \\
& =q_{k}^{* T} q^{*}+u_{k}^{* T} \bar{u}_{k}+w_{k}^{T} \nabla \mathscr{L}_{\lambda_{k}}\left(p_{k}, x_{k}\right)\left(q^{*}, \bar{u}_{k}\right) \leq 0
\end{aligned}
$$

by means of (5.34). By passing $k$ to infinity we obtain $q^{* T} q^{*} \leq 0$ implying $q^{*}=0$. Thus all assumptions of Theorem 3.5 are fulfilled and the statement is established.

In case when $P=\mathbb{R}^{l}$ Theorem 6.1 improves [18, Theorem 6] by weakening the assumption that the multiplier $\lambda \in N_{D}(\tilde{g}(p, x))$ satisfying $b(\bar{p}, \bar{x})^{T} \lambda=-f(\bar{p}, \bar{x})$ is unique.

Example 6.2 It is easy to see that for the variational system of Example 5.5 the solution map $S$ given by (5.37) has the Aubin property relative to its domain $\operatorname{dom} S=\left\{\left(p_{1}, p_{2}\right)\right.$ | $\left.p_{2}-p_{1} \leq 0, p_{2}-2 p_{1} \leq 0\right\}$. We want now to analyze the conditions on the set $P$ provided by Theorem 6.1 such that $S$ has the Aubin property relative to $P$. After some calculations we obtain the following table where we list all directions $(q, u)$ such that (6.39) holds as well as $\hat{\lambda} \in \Xi((\bar{p}, \bar{x}),-f(\bar{p}, \bar{x}) ;(q, u))$ and $\eta \in N_{\mathscr{K}_{D}(\tilde{g}(\bar{p}, \bar{x}), \hat{\lambda})}(\nabla \tilde{g}(\bar{p}, \bar{x})(q, u))$ such that $0=\nabla \mathscr{L}_{\hat{\lambda}}(\bar{p}, \bar{x})(q, u)+b(\bar{p}, \bar{x})^{T} \eta$. In addition we display vector $\nabla \tilde{g}(\bar{p}, \bar{x})(q, u)$, useful also for the computation of $\eta$.

\begin{tabular}{|c|c|c|c|c|}
\hline$q$ & $u$ & $\hat{\lambda}$ & $(\nabla \tilde{g}(\bar{p}, \bar{x})(q, u))^{T}$ & $\eta \in \mathbb{R}_{+}^{2}$ \\
\hline$q_{1} \geq 0, q_{2}-q_{1} \leq 0$ & $\left(q_{1}, 0\right)$ & 0 & $\left(q_{2}-q_{1},-q_{1}\right)$ & $(0,0)$ \\
$q_{2}-q_{1} \leq 0, q_{2}-2 q_{1}<0$ & $\left(q_{1}, \frac{q_{1}-q_{2}}{2}\right)$ & 0 & $\left(0, q_{2}-2 q_{1}\right)$ & $\left(\frac{q_{1}-q_{2}}{2}, 0\right)$ \\
$q_{1} \leq 0, q_{2}=2 q_{1}$ & $\left(q_{1}, \frac{-q_{1}}{2}\right)$ & 0 & $(0,0)$ & $\eta_{1}+\eta_{2}=\frac{-q_{1}}{2}$ \\
$q_{1} \leq 0, q_{2}-2 q_{1}<0$ & $\left(q_{1}, \frac{-q_{1}}{2}\right)$ & 0 & $\left(q_{2}-2 q_{1}, 0\right)$ & $\left(\frac{-q_{1}}{2}, 0\right)$ \\
\hline
\end{tabular}

From this table we see that condition (i) of Theorem 6.1 amounts to the requirement that

$$
T_{P}(\bar{p}) \subset\left\{q \in \mathbb{R}^{2} \mid q_{2}-q_{1} \leq 0, q_{2}-2 q_{1} \leq 0\right\}(=\operatorname{dom} S) .
$$

In the next step we will analyze condition (ii) of Theorem 6.1. Since $\nabla_{2} \tilde{g}(\bar{p}, \bar{x})$ has full rank, implication (6.40) holds for any direction $(q, u)$. Consider now $(0,0) \neq(q, u)$ together with $\hat{\lambda}=0$ and $\eta$ from table (6.42) and faces $\mathscr{F}_{1}, \mathscr{F}_{2}$ of the critical cone $\mathscr{K}_{D}(\tilde{g}(\bar{p}, \bar{x}), \hat{\lambda})=\mathbb{R}_{-}^{2}$ satisfying $\nabla \tilde{g}(\bar{p}, \bar{x})(q, u) \subset \mathscr{F}_{2} \subset \mathscr{F}_{1} \subset[\eta]^{\perp}$. Observe that $(q, u) \neq(0,0)$ implies $q \neq 0$. Further, consider $0 \neq w \in \mathbb{R}^{2}$ such that $b(\bar{p}, \bar{x}) w=$ $\left(w_{2}, w_{2}\right)^{T} \in \mathscr{F}_{1}-\mathscr{F}_{2}$. It follows that $w_{2}=0$ whenever $\eta \neq 0$. Further, $\mathscr{F}_{1}=\mathbb{R}_{-}^{2}$ when $w_{2} \neq 0$ and $\mathscr{F}_{1}-\mathscr{F}_{2}=\mathbb{R}^{2}$ if $w_{2}>0$. Our next analysis is split into three cases.

Case w2 $>$ 0: $\quad$ Then $\mathscr{F}_{1}-\mathscr{F}_{2}=\mathbb{R}^{2}$ and obviously $\tilde{w}=\left(0,-w_{2}\right)$ fulfills $\nabla_{2} \tilde{g}(\bar{p}, \bar{x}) \tilde{w} \in$ $\mathbb{R}^{2}$ and

$$
w^{T} \nabla_{2} \mathscr{L}_{\hat{\lambda}}(\bar{p}, \bar{x}) \tilde{w}=w_{2}^{2}>0 .
$$

Case $\mathbf{w}_{2}<\mathbf{0}$ : It follows that $\eta=0$ and $\mathscr{F}_{1}=\mathbb{R}_{-}^{2}$. If $\nabla \tilde{g}_{1}(\bar{p}, \bar{x})(q, u)<0$ then $\mathscr{F}_{1}-\mathscr{F}_{2} \supset \mathbb{R} \times \mathbb{R}_{-}$and we can take $\tilde{w}=\left(0,-w_{2}\right)$ to obtain $\nabla_{2} \tilde{g}(\bar{p}, \bar{x}) \tilde{w}=$ $\left(-2 w_{2}, 2 w_{2}\right)^{T} \in \mathbb{R} \times \mathbb{R}_{-} \subset \mathscr{F}_{1}-\mathscr{F}_{2}$ and $w^{T} \nabla_{2} \mathscr{L}_{\hat{\lambda}}(\bar{p}, \bar{x}) \tilde{w}=w_{2}^{2}>0$. Hence assume that $\nabla \tilde{g}_{1}(\bar{p}, \bar{x})(q, u)=0$. A look at table (6.42) tells us that this together with $\eta=0$ and $q \neq 0$ is only possible when $q_{2}=q_{1}$ and $q_{1}>0$. In this case we can take $w=(-1,-2)$ and $\mathscr{F}_{2}=\{0\} \times \mathbb{R}_{-}, \mathscr{F}_{1}=\mathbb{R}_{-}^{2}$ resulting in $\mathscr{F}_{1}-\mathscr{F}_{2}=\mathbb{R}_{-} \times \mathbb{R}$ and it follows that there does not exist any

$$
\tilde{w} \in\left\{\tilde{w} \mid \nabla_{2} \tilde{g}(\bar{p}, \bar{x}) \tilde{w} \in \mathscr{F}_{1}-\mathscr{F}_{2}\right\}=\left\{\tilde{w} \mid-\tilde{w}_{1}+2 \tilde{w}_{2} \leq 0\right\}
$$


fulfilling

$$
w^{T} \nabla_{2} \mathscr{L}_{\hat{\lambda}}(\bar{p}, \bar{x}) \tilde{w}=-\tilde{w}_{1}+2 \tilde{w}_{2}>0 .
$$

Case $\mathbf{w}_{2}=\mathbf{0}: \quad$ Note that $w \neq 0$ implies $w_{1} \neq 0$. If $\nabla \tilde{g}_{1}(\bar{p}, \bar{x})(q, u)<0$ then $\mathscr{F}_{1}-\mathscr{F}_{2} \supset$ $\mathbb{R} \times\{0\}$ and we can take $\tilde{w}=\left(w_{1}, w_{1} / 2\right)$ to obtain $\nabla_{2} \tilde{g}(\bar{p}, \bar{x}) \tilde{w} \in\{0\} \times \mathbb{R} \subset \mathscr{F}_{1}-\mathscr{F}_{2}$ and $w^{T} \nabla_{2} \mathscr{L}_{\hat{\lambda}}(\bar{p}, \bar{x}) \tilde{w}=w_{1}^{2}>0$. If $\nabla \tilde{g}_{2}(\bar{p}, \bar{x})(q, u)<0$, then we can argue as before to show that $\tilde{w}=\left(w_{1},-w_{1} / 2\right)$ fulfills the required conditions. There remains the case that $\nabla \tilde{g}(\bar{p}, \bar{x})(q, u)=(0,0)$. A look at Table 6.42 shows that this is possible for nonzero $q$ only in case when $q_{2}=2 q_{1}$ and $q_{1}<0$. Taking $\eta=\left(-q_{1} / 2,0\right), \mathscr{F}_{2}=\mathscr{F}_{1}=\{(0,0)\}$, we obtain that the only $\tilde{w}$ with $\nabla_{2} \tilde{g}(\bar{p}, \bar{x}) \tilde{w} \in \mathscr{F}_{1}-\mathscr{F}_{2}$ is $\tilde{w}=(0,0)$ and therefore we again cannot fulfill the condition $w^{T} \nabla_{2} \mathscr{L}_{\hat{\lambda}}(\bar{p}, \bar{x}) \tilde{w}>0$.

The above analysis shows that we have to exclude the sets $P$ such that

$$
T_{P}((0,0)) \cap\{(t, t),(-t,-2 t) \mid t>0\} \neq \emptyset .
$$

This means that, by virtue of Theorem 6.1, $S$ has the Aubin property relative to $P$ around $(\bar{p}, \bar{x})$ for every closed set $P$ containing $(0,0)$ such that

$$
T_{P}((0,0)) \subset\left\{q \in \mathbb{R}^{2} \mid q_{2}-q_{1} \leq 0, q_{2}-2 q_{1} \leq 0\right\} \backslash\{(t, t),(-t,-2 t) \mid t>0\}(=\operatorname{int} \operatorname{dom} S \cup\{(0,0)\}) .
$$

\section{Conclusion}

In most rules of generalized differentiation one associates with the data a certain mapping and requires, as a qualification condition, the metric subregularity of this mapping at the considered point, see, e.g., [20, 22-24]. Correspondingly, in the directional limiting calculus the qualification conditions amount typically to the directional metric subregularity of the respective associated mappings, cf. [1]. In both cases, however, the required property should be verifiable via suitable conditions expressed solely in terms of problem data. In this paper we construct such conditions on the basis of the (stronger) property of directional metric regularity, see Theorems $2.11,3.5,5.3$ and 6.1 .

In general, the principal questions related to metric subregularity, calmness and the associated areas of error bounds and subtransversality have been thoroughly investigated by many notable researchers including A. Y. Kruger $([6-8,26]$ and many other works on this subject). Via the research, discussed in this paper, the authors would like to give credit to their friend Alex on the occasion of his $65^{\text {th }}$ birthday.

Acknowledgements The research of the first two authors was supported by the Austrian Science Fund (FWF) under grant P29190-N32. The research of the third author was supported by the Grant Agency of the Czech Republic, Project 17-08182S and the Australian Research Council, Project DP160100854.

Funding Information Open access funding provided by Austrian Science Fund (FWF).

Open Access This article is distributed under the terms of the Creative Commons Attribution 4.0 International License (http://creativecommons.org/licenses/by/4.0/), which permits unrestricted use, distribution, and reproduction in any medium, provided you give appropriate credit to the original author(s) and the source, provide a link to the Creative Commons license, and indicate if changes were made. 


\section{References}

1. Benko, M., Gfrerer, H., Outrata, J.V.: Calculus for directional limiting normal cones and subdifferentials, Set-Valued Var. Anal., https://doi.org/10.1007/s 11 228-018-04+2-5

2. Baiocchi, C., Capelo, A.: Variational and quasivariational inequalities. Applications to free boundary problems. Wiley, New York (1984)

3. Bonnans, J.F., Shapiro, A.: Perturbation analysis of optimization problems. Springer, New York (2000)

4. Dontchev, A.L., Rockafellar, R.T.: Regularity and conditioning of solution mappings in variatonal analysis. Set-Valued Anal. 12, 79-109 (2004)

5. Dontchev, A.L., Rockafellar, R.T.: Implicit functions and solution mappings. Springer, Heidelberg (2014)

6. Dontchev, A.L., Gfrerer, H., Kruger, A.Y., Outrata, J.V.: The radius of metric subregularity, submitted, arXiv: 1807.02198

7. Fabian, M., Henrion, R., Kruger, A.Y., Outrata, J.V.: Error bounds: necessary and sufficient conditions. Set-Valued Var. Anal. 18, 121-149 (2010)

8. Fabian, M., Henrion, R., Kruger, A.Y., Outrata, J.V.: About error bounds in metric spaces. In: Klatte, D., Lüthi, H.-J., Schmedders, K. (eds.) Operation Research Proceedings 2011. Springer, Berlin (2012)

9. Gfrerer, H.: First order and second order characterizations of metric subregularity and calmness of constraint set mappings. SIAM J. Optim. 21, 1439-1474 (2011)

10. Gfrerer, H.: On directional metric regularity, subregularity and optimality conditions for nonsmooth mathematical programs. Set-Valued Var. Anal. 21, 151-176 (2013)

11. Gfrerer, H.: On directional metric subregularity and second-order optimality conditions for a class of nonsmooth mathematical programs. SIAM J. Optim. 23, 632-665 (2013)

12. Gfrerer, H.: On metric pseudo-(sub)regularity of multifunctions and optimality conditions for degenerated mathematical programs. Set-Valued Var. Anal. 22, 79-115 (2014)

13. Gfrerer, H., Klatte, D.: Lipschitz and Hölder stability of optimization problems and generalized equations. Math. Program. Ser. A 158, 35-75 (2016)

14. Gfrerer, H., Mordukhovich, B.S.: Robinson stability of parametric constraint systems via variational analysis. SIAM J. Optim. 27, 438-465 (2017)

15. Gfrerer, H., Mordukhovich, B.S.: Second-order variational analysis of parametric constraint and variational systems, (2017), to appear in SIAM. J. Optim., arXiv:1711.07082

16. Gfrerer, H., Outrata, J.V.: On Lipschitzian properties of implicit multifunctions. SIAM J. Optim. 26, 2160-2189 (2016)

17. Gfrerer, H., Outrata, J.V.: On the Aubin property of a class of parameterized variational systems. Math. Methods Oper. Res. 86, 443-467 (2017)

18. Gfrerer, H., Outrata, J.V.: On the Aubin property of solution maps to parameterized variational systems with implicit constraints, (2018), submitted, arXiv:1810.12604

19. Ginchev, I., Mordukhovich, B.S.: On directionally dependent subdifferentials. C.R. Bulg. Acad. Sci. 64, 497-508 (2011)

20. Henrion, R., Jourani, A., Outrata, J.V.: On the calmness of a class of multifunctions. SIAM J. Optim. 13, 603-618 (2002)

21. Henrion, R., Outrata, J.V.: Calmness of constraint systems with applications. Math. Programming, Ser. B 104, 437-464 (2005)

22. Ioffe, A.D.: Necessary and sufficient conditions for a local minimum 1: a reduction theorem and first order conditions. SIAM J. Control Optim. 17, 245-250 (1979)

23. Ioffe, A.D.: Regular points of Lipschitz functions. Trans. Amer. Math Soc. 251, 61-69 (1979)

24. Ioffe, A.D., Outrata, J.V.: On metric and calmness qualification conditions in subdifferential calculus. Set-Valued Anal. 16, 199-227 (2008)

25. Klatte, D., Kummer, B.: Nonsmooth equations in optimization. Regularity, calculus, methods and applications, Nonconvex optimization and its applications, vol. 60. Kluwer Academic Publishers, Dordrecht (2002)

26. Kruger, A.Y.: Error bounds and metric subregularity. Optimization 64, 49-79 (2015)

27. Levy, A.B.: Implicit multifunction theorems for the sensitivity analysis of variational conditons. Math Program. 74, 333-350 (1996)

28. Mordukhovich, B.S.: Variational analysis and generalized differentiation, vol I: basic theory. Springer, Berlin (2006)

29. Mordukhovich, B.S., Outrata, J.V.: Coderivative analysis of quasi-variational inequalities with applications to stability and optimization. SIAM J. Optim. 18, 389-412 (2007)

30. Mordukhovich, B.S.: Variational analysis and applications. Springer, Cham (2018) 
31. Robinson, S.M.: Stability theory for systems of inequalities, II: differentiable nonlinear systems. SIAM J. Numer. Anal. 13, 497-513 (1976)

32. Robinson, S.M.: Constraint nondegeneracy in variational analysis. Math. of Oper. Res. 28, 201-232 (2003)

33. Rockafellar, R.T.: Convex analysis. Princeton, New Jersey (1970)

34. Rockafellar, R.T., Wets, R.J.-B.: Variational analysis. Springer, Berlin (1998)

35. Zheng, X.Y., Ng, K.F.: Metric subregularity and calmness for nonconvex generalized equations in Banach spaces. SIAM J. Optim. 20, 2119-2136 (2010)

Publisher's Note Springer Nature remains neutral with regard to jurisdictional claims in published maps and institutional affiliations. 